Gabriele Schmidhuber

\title{
Dionysische Frauen in der griechischen Vasenmalerei: Nymphen oder Mänaden?*
}

\section{Die literarische Überlieferung}

Schon bei Homer ist die Vorstellung von Nymphen mit ihren ausschlaggebenden Charaktereigenschaften

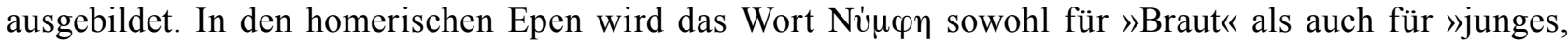
heiratsfähiges Mädchen« und gelegentlich für bereits verheiratete Frauen verwendet, meistens jedoch sind mit der Bezeichnung weibliche Landschafts- oder Wassergottheiten gemeint ${ }^{1}$. Die Etymologie des Wortes ist unbekannt, wahrscheinlich gibt es aber einen Bezug zum Lateinischen nubo (ich heirate) ${ }^{2}$.

Einerseits sind Nymphen Naturgottheiten, die Gewässer, Grotten, Berge und Auen bewohnen, andererseits wird die Gruppe der nysäischen Nymphen, der Nymphen von Nysa, bereits in der »llias « mit Dionysos verbunden, wo sie die Ammen des Dionysosknaben sind ${ }^{3}$. Hier wird auch erwähnt, dass sie ihre $\theta \dot{v} \sigma \theta \lambda \alpha$ wegwerfen, die wohl eine Vorform der Thyrosstäbe darstellen, die im Dionysoskult später zu einem spezifischen Attribut werden ${ }^{4}$. Eine weitere literarische Quelle von Bedeutung ist der homerische Aphroditehymnos, in welchem Nymphen und Silene erstmals miteinander in Verbindung gebracht werden ${ }^{5}$. Im Hymnus an Dionysos wird abermals erwähnt, dass die Nymphen von Nysa das Dionysoskind nähren und der erwachsene Weingott später der Führer der Göttinnen wird, mit welchen er gemeinsam durch die Wälder zieht ${ }^{6}$.

Im Drama der Klassik werden die Frauen, welche sich mit Dionysos im ekstatischen Treiben befinden, meist als Mänaden (»Rasende«) bezeichnet. Für diesen Begriff gibt es in der attischen Tragödie zwei Synonyme, nämlich Bakche oder Bakchantin (»die dem Bakchos«) und Thyiade (»die Dahinstürmende«). Euripides nennt in seinen >Bakchen Sie werden mit Wahnsinn bestraft und müssen folglich als rasende Mänaden in den Wäldern leben?

Von großer Bedeutung für die Interpretation der Vasenbilder ist die Unterscheidung zwischen positiver und negativer $\mu \alpha v i ́ \alpha$, die Euripides konstatierte: Der asiatische Bakchenchor erfährt eine Form der dionysischen

\footnotetext{
* Der Artikel basiert auf den Ergebnissen meiner Diplomarbeit, die ich im Herbst 2005 unter der Betreuung von Prof. Florens Felten an der Universität Salzburg eingereicht habe. Einen großen Teil der Arbeit konnte ich während eines dreimonatigen Aufenthaltes am ÖAI Athen bewältigen, an welchem ich wohnen und arbeiten durfte, wofür ich mich an dieser Stelle herzlich bedanken möchte. - Die Diplomarbeit beschäftigt sich mit der ikonographischen Entwicklung von Nymphendarstellungen in der griechischen Vasenmalerei vom 7. bis zum 4. Jh. v. Chr. Das größte Problem hierbei stellt die Unterscheidung von Nymphen und Mänaden dar, weshalb das besondere Augenmerk der Abhandlung darauf gerichtet ist, eine Charakterisierung der dionysischen Frauenfiguren vorzunehmen und darauf aufbauend Kriterien für deren Benennung zu finden. - Die Abkürzungen folgen den Richtlinien des Österreichischen Archäologischen Instituts $<$ http://www.oeai.at/publik/autoren.html $>$, weitere hier verwendete Kurzzitate finden sich am Ende des Beitrags.

${ }^{1}$ P. Reinbacher, Die Personifikation von Städten und Ländern in der griechischen Kunst unter besonderer Berücksichtigung der Nymphen als Ortsrepräsentanten (Mag. Universität Graz 1990) 18.

2 Larson 2001, 281 Anm. 1; vgl. V. Andò, Nymphe: La sposa e le Ninfe, QuadUrbin 52, 1996, 47-49.

3 Hom. Il. 6, 132-134.

${ }^{4}$ Larson 2001, 93.

${ }^{5}$ Hom. h. Aphr. 257-263.

${ }^{6}$ Hom. h. Dion. 4-10.

7 Moraw 1998, 15-17.
} 


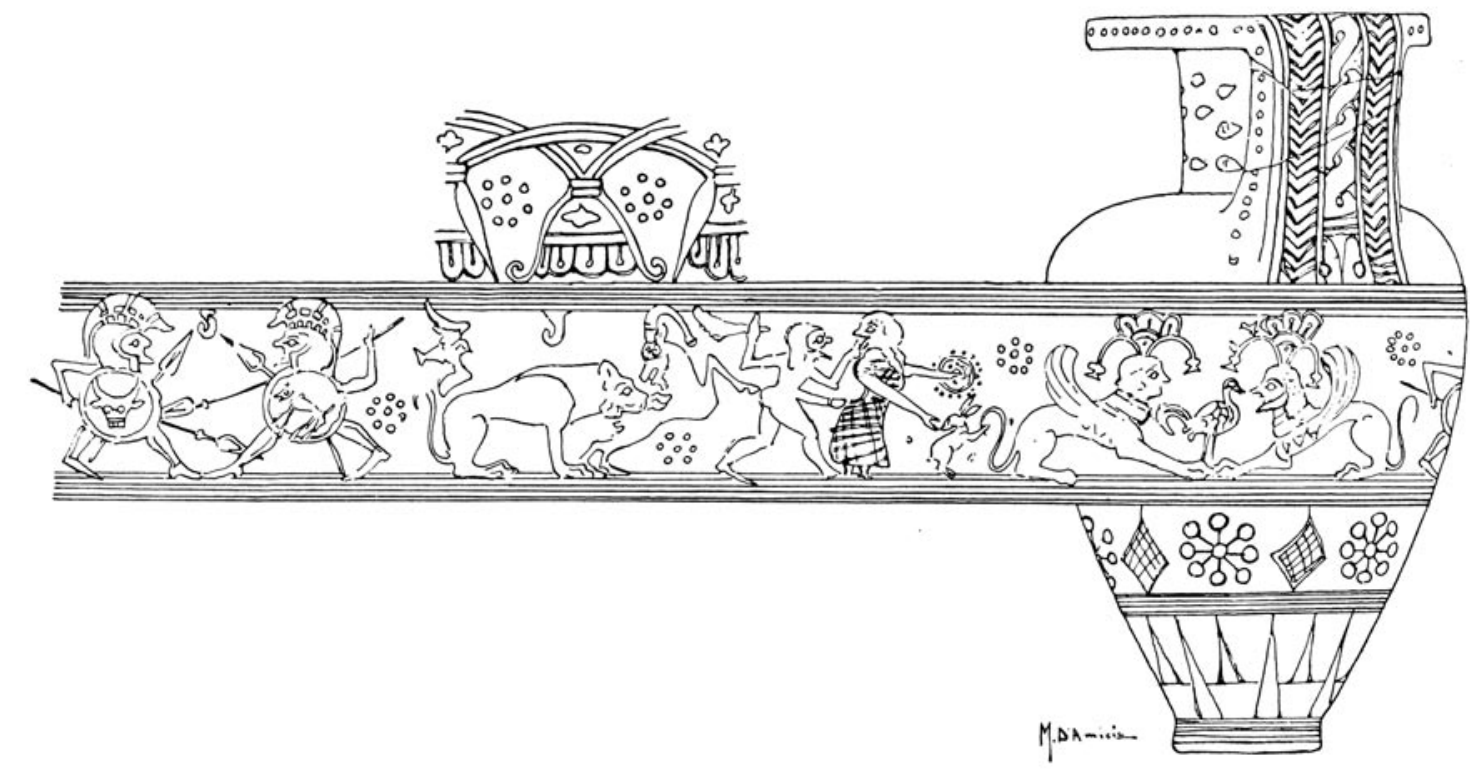

1 SF 1: Spitzaryballos, Brindisi 1669

Gruppenekstase (positiv), wohingegen die thebanischen Frauen mit destruktivem Wahnsinn geschlagen werden (negativ) und infolgedessen Pentheus schließlich töten ${ }^{8}$.

Die Nymphen oder göttlichen Ammen werden also in der klassischen Literatur meist als Mänaden bezeichnet, wobei Begriffe des Mythos lediglich in Begriffe des Kultes umgewandelt wurden, der Frauentyp jedoch derselbe bleibt ${ }^{9}$. Der wichtige Unterschied zwischen Nymphen und Mänaden bezieht sich darauf, dass Mänaden sterbliche Frauen sind, die vorübergehend für einen bestimmten Zeitraum als rasende Begleiterinnen des Gottes leben, während Nymphen göttliche und sehr langlebige ${ }^{10}$ Wesen sind.

\section{Die Darstellungen in der schwarzfigurigen Vasenmalerei}

Die frühen Darstellungen vom 7. Jahrhundert v. Chr. bis ca. 580 v. Chr.

Um die Mitte des 7. Jahrhunderts v. Chr. findet sich auf einem spät-protokorinthischen Spitzaryballos (SF 1, Abb. 1) ${ }^{11}$ wohl die früheste Darstellung eines Vorläufers eines Silens und seiner Begleiterin. Ein ityphallischer, bärtiger Mann geht mit einer Keule auf eine Frau los, welche in ein langes, verziertes Gewand gehüllt ist, in ihrer rechten Hand einen Kranz hält und mit ihrer Linken auf einen Hasen zeigt. Dieses Tier wird als Spielzeug und Wild angesehen und kehrt in den Werken des Lydos und des Amasis-Malers als Attribut der dionysischen Frauen wieder ${ }^{12}$. Im ersten Viertel des 6. Jahrhunderts v. Chr. folgen attische Darstellungen, die derartige angriffslustige Verfolgungsszenen zeigen, z. B. die bekannte Lekythos in der Art des GorgoMalers (SF 2, Abb. 2). Hier wird erstmals ein zur Gänze ausgebildeter Silen bei der Verfolgung einer Frau abgebildet. Er reitet auf einem Maultier heran, die Frau ist laufend dargestellt, sodass ihr linkes Bein vom langen Gewand entblößt ist. Die Frau wird von dem Maultier in den rechten Oberarm gebissen, wodurch

\footnotetext{
${ }^{8}$ Henrichs 1994, 34-38.

${ }^{9}$ Moraw 1998, $17 \mathrm{f}$.

${ }^{10}$ Vgl. Hes. fr. 304 (Zählung der Fragmente nach der Ausgabe von R. Merkelbach - M. L. West, Fragmenta Hesiodea [Oxford 1967]).

${ }^{11}$ Die arabischen Ziffern in Klammer beziehen sich auf den Katalog, wobei die Bilder in schwarzfigurig (SF) und rotfigurig (RF) aufgeteilt werden.

${ }^{12}$ Isler-Kerényi 1991, 296 f.
} 
die Aggressivität der Verfolgung verstärkt zum Ausdruck gebracht wird ${ }^{13}$.

Aufgrund der literarischen Überlieferung kann man davon ausgehen, dass die Partnerinnen der Silene Nymphen sind, so wie es im Aphroditehymnus beschrieben wird, auch wenn den Frauen in dieser frühen Phase noch keine spezifisch dionysischen Attribute beigegeben sind ${ }^{14}$. Die Silene werden in der Frühzeit als unzivilisierte Naturwesen charakterisiert und oftmals bei der aggressiven Verfolgung von Frauen dargestellt, die ebenfalls in die wilde Natur gehören, wir ihre Bewegungen, entblößten Körperteile oder kurzen Gewänder und Wurfgegenstände zeigen ${ }^{15}$. Die Entblößung der Beine enthält auch eine erotische Komponente (wie auch bei tanzenden Komasten und Hetären zu beobachten), wodurch die sexuelle Beziehung zwischen Nymphen und Silenen bereits angedeutet wird ${ }^{16}$.

Die ersten Nymphen im Gefolge des Dionysos

Erstmals im dionysischen Gefolge anzutreffen und auch inschriftlich als solche gesichert sind Nymphen auf Gefäßen des Sophilos und des Kleitias: Zwei Dinoi des Sophilos zeigen jeweils eine Prozession zur Hochzeit von Peleus und Thetis. Am Londoner Dinos (SF 3) folgen drei als NYФAI bezeichnete Frauen nach Hebe, Chiron und Themis dem Weingott Dionysos. Auf einem Fragment des Athener Dinos (SF 4, Abb. 3) sind drei Frauenköpfe zu sehen, wobei die linke Frau en face abgebildet ist und die Syrinx spielt. Die Dargestellten tragen die Beischrift NY $\Sigma A I^{17}$. J. D. Beazley verbindet das Wort mit den Nymphen von Nysa, welche den Dionysosknaben aufziehen ${ }^{18}$. Die Frauen unterscheiden sich in ihrer Darstellungsart nicht von den restlichen Göttinnen der festlichen Züge. Sie tragen ebenso lange Haare, die hinter die Ohren gestrichen werden und auf die Schul-

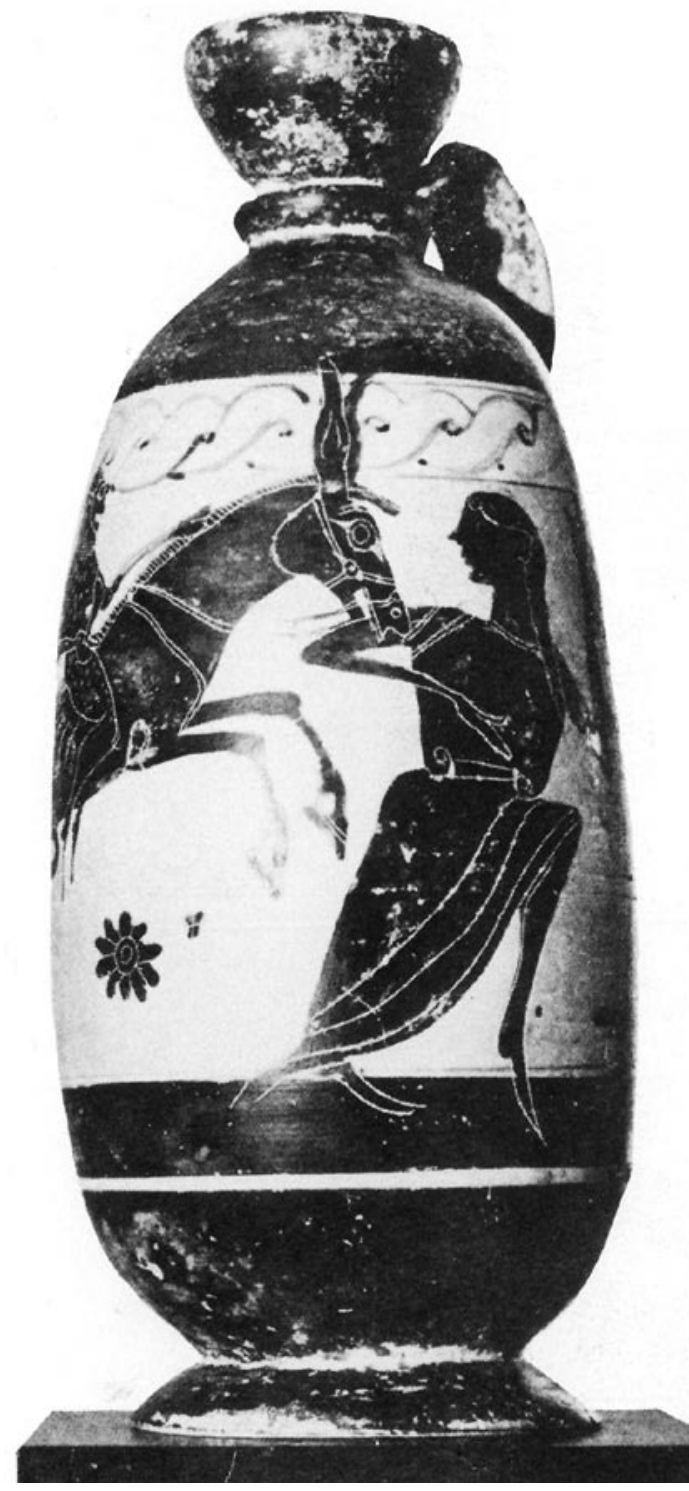

2 SF 2: Lekythos, Buffalo, Albright Gallery G 600 tern herabfallen. Haarreifen, Schmuck und reich verzierte Gewänder sind teilweise auszumachen. Zudem ist die Syrinx ein bemerkenswertes Attribut der Nymphen, denn im 6. Jahrhundert v. Chr. wird häufig Hermes und später auch Pan mit diesem Instrument dargestellt. Somit soll die Syrinx als Beiwerk der nysäischen Nymphen wohl die Verbindung zu den ländlichen Gottheiten und der Natur ausdrücken. Darüber hinaus ist festzuhalten, dass auf den Vasen des Sophilos die Nymphen erstmals nicht in Begeleitung der Silene, sondern in Verbindung mit Dionysos erscheinen ${ }^{19}$.

\footnotetext{
${ }^{13}$ Moraw 1998, 31; Isler-Kerényi 1991, 296.

${ }^{14}$ Hom. h. Aphr. 257-263.

15 Isler-Kerényi 1999, 556; Moraw 1998, 31.

${ }_{16}$ Moraw 1998, $32 \mathrm{f}$.

${ }^{17}$ Schöne 1987, $20 \mathrm{f}$.

18 J. D. Beazley, The development of Attic black-figure (Berkeley 1986) 17. T. H. Carpenter hält das Wort »Nysai« hingegen für

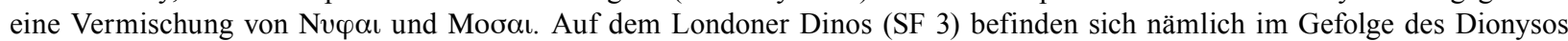
auch die Musen (mit Beischrift), deren Darstellung jener der Nvoou des Athener Dinos annähernd gleicht: Carpenter 1986, 9.

19 Moraw 1998, 34 f.
} 


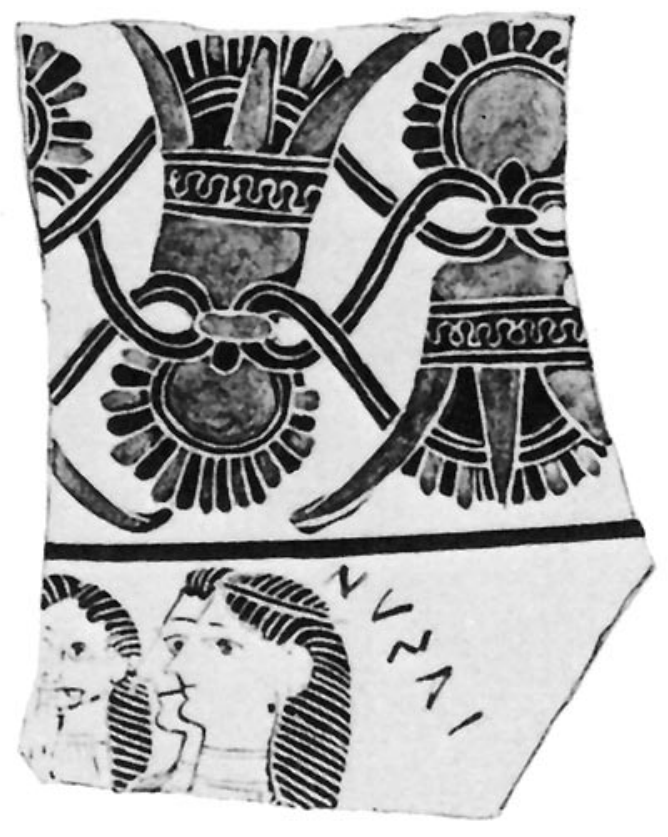

3 SF 4: Dinos, Athen, Nationalmuseum 15165

Die Beischrift NYФAI begegnet uns abermals am sog. Françoisvase des Kleitias (SF 5) bei der Rückführung des Hephaistos in den Olymp. Dionysos folgen zuerst Hephaistos auf einem Maultier und anschließend mehrere Silene nach. Sie tragen die Beischrift $\Sigma$ I $\Lambda$ ENOI; der dritte Silen trägt eine Nymphe im Arm. Die letzte Nymphe hält Krotalen (Kymbala), also dionysische Schlaginstrumente, in Händen. Die Frauen tragen lange, gegürtete Peploi mit kurzem Überschlag, der mit einer Art Karomuster verziert ist. Das Gebäude des Brunnenhauses verbindet die dionysische Szene mit der Troilosepisode. Die Nymphen kommen demnach vom Brunnenhaus her, dem wohl geeignetsten Ort für Naturgottheiten ${ }^{20}$. Nymphen und Silene befinden sich hier erstmals gemeinsam im dionysischen Gefolge, wobei die Verbindung durch die Nymphe, die sich in den Armen des Silens befindet, besonders deutlich wird. Die anderen Nymphen musizieren, wahrscheinlich tanzen sie auch ${ }^{21}$. Kleitias hat somit das Gefolge des Dionysos komplettiert, indem er neben den Nymphen zum ersten Mal auch Silene abbildet, und legte hiermit den Keim für den mythischen Thiasos, als die Silene jetzt Attribute wie Wein, Musik und Frauen erhalten ${ }^{22}$. Die überlieferten Vasenbilder zeigen, dass Nymphen und Silene anfangs unabhängig von Dionysos auftreten können ${ }^{23}$, dann die Nymphen und schließlich diese gemeinsam mit den Silenen in den Thiasos eintreten ${ }^{24}$. Am `Kleitiaskrater wird die Verschmelzung dieser beiden Entwicklungslinien deutlich: erstens die Nymphen als Gefährtinnen der Silene und zweitens die Nymphen im Gefolge des Dionysos (vielleicht die Nymphen von Nysa) ${ }^{25}$.

In der ersten Hälfte des 6. Jahrhunderts v. Chr. ändert sich folglich das Verhältnis zwischen Nymphen und ihren Begleitern: Zuerst werden die Frauen von wilden, aggressiven und unzivilisierten Silenen verfolgt. Später, beginnend mit dem >Kleitiaskrater〈, entwickelt sich durch die Einbindung in den Thiasos und den gemeinsamen Tanz ein gewaltfreies Verhältnis dieser Naturwesen, sodass die Nymphen das weibliche Pendant zu den Silenen darstellen und sie mit diesen gemeinsam im Dienst des Gottes Dionysos stehen ${ }^{26}$.

\section{Die Beigabe dionysischer Attribute}

In der Folgezeit, nämlich in den Jahren zwischen 560 und 530 v. Chr., nimmt die Zahl der Bilder mit dionysischem Kontext stark zu und den Nymphen werden erstmals spezifisch dionysische Attribute beigefügt. Auf dem Kolonettenkrater des Lydos in New York (SF 6, Abb. 4), der wiederum die Rückführung des

${ }^{20}$ Schöne 1987, 18-20; Isler-Kerényi 1999, 557 f.

${ }^{21}$ Moraw 1998, 36.

22 McNally 1978, 108.

${ }^{23}$ Allerdings ist es fraglich, ob manche dieser Vasen nicht doch in den dionysischen Kontext gehören: Beispielsweise zeigt die Lekythos in Buffalo (SF 2) den Silen auf einem Maultier, welches zumindest seit dem >Kleitiaskrater die gesamte Antike hindurch zur Sphäre des Dionysos zählt. Vgl. dazu Schöne 1987, 21; Isler-Kerényi 1999, 556.

${ }^{24}$ Larson 2001, 93.

${ }_{25}$ Moraw 1998, $36 \mathrm{f}$.

${ }^{26}$ Isler-Kerényi 1991, 297; Moraw 1998, 103; Carpenter 1986, 85. Dazu ist zu bemerken, dass sich die Ikonographie des Thiasos aus dem Komos entwickelte. Um 600 v. Chr. übernahmen attische Maler korinthische Vorbilder. In Athen werden die männlichen Komasten meist von nackten oder kurz gewandeten Frauen begleitet, wodurch die freizügige Sexualität dieser Frauen, welche als Hetären oder Gefährtinnen bezeichnet werden, betont wird. In dem Moment, in dem der Komos zum Thiasos wird, verwandeln sich die Männer in Silene, die Frauen verändern sich äußerlich nicht. Die Nymphen sind also die in der menschlichen Sphäre begleitenden Hetären des Symposions, was durch die sexuelle Beziehung zu den Silenen bestärkt wird. Vgl. dazu Isler-Kerényi 1991, 295; Moraw 1998, 37-39; Isler-Kerényi 1999, 559-562. 


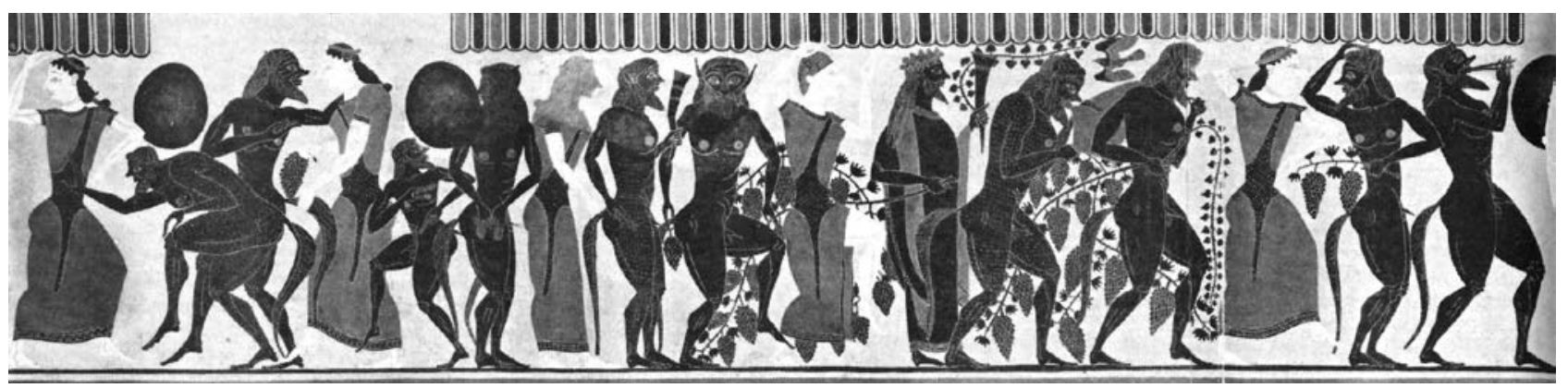

4 SF 6: Kolonettenkrater, New York, MMA 31.11.11

Hephaistos thematisiert, haben die Figuren des Thiasos eine monumentale Größe erreicht und umziehen das gesamte Gefäß ${ }^{27}$. Lydos beginnt als erster Maler, viele verschiedene Attribute zu verwenden: So spielen hier Silene den Doppelaulos, tragen Weinschläuche, halten Efeuzweige und Weinreben. Efeu und Wein waren bisher Dionysos vorbehalten, durch die Übertragung auf das Gefolge wird die Verbundenheit zum Gott betont. Die Nymphen tragen über ihrem langen Peplos ein Fell, das wahrscheinlich die Nebris (Rehkalbfell) meint; eine hat eine bärtige Schlange um ihre Taille gelegt. Die Ausstattung mit Tierfellen wird in der Folgezeit zum Usus, wobei die Felle als Schutz dienen, zugleich das kämpferische, wehrhafte Wesen der Frauen betonen und deren Naturnähe zeigen. Durch die Schlange werden die Beziehung zu Dionysos, welcher auf Gigantomachiedarstellungen mit Schlangen erscheint, sowie die Beherrschung der Tiere hervorgehoben ${ }^{28}$. Auf dem New Yorker Krater stehen Silene und Nymphen nicht beziehungslos nebeneinander, sondern erstmals legt ein Silen den Arm um sein weibliches Pendant, während die Nymphe an seinem Pferdeschwanz zieht ${ }^{29}$.

Der Amasis-Maler hat als einer der Ersten zahlreiche seiner Bildthemen dem dionysischen Geschehen gewidmet. Auf einer Halsamphora in Paris (SF 7) treten Nymphen beispielsweise ohne männliche Begleitung auf: Vor dem Weingott tanzen zwei eng umschlungene Frauen, welche Schmuck und Efeukränze tragen und Efeuzweige in den Händen halten. Die vordere trägt ein Pantherfell über ihrem Peplos (Pardalis) und hält in ihrer Linken einen kleinen Hirsch, während die andere Dionysos einen Hasen entgegenstreckt ${ }^{30}$. Die Halsamphora zeigt, dass die dionysischen Frauen ab der Mitte des 6. Jahrhunderts v. Chr. durch festgelegte Attribute eine Identität und zentrale Bedeutung im Dionysoskult erlangt haben und als solche identifiziert werden können, auch wenn sie in andere Bildkontexte eingefügt werden ${ }^{31}$.

Es entwickelt sich ein freundschaftliches Verhältnis, wodurch sich Silene und Nymphen auch körperlich näherkommen. Mit Ausnahme des `Kleitiaskraters`, der wohl erstmals ein nicht aggressives Verhältnis zeigt, kam es auf früheren Vasen nur bei Verfolgungs- und Entführungsszenen zu Berührungen ${ }^{32}$. In den Jahren zwischen 560 und $530 \mathrm{v}$. Chr. wird die Verbindung des dionysischen Gefolges immer enger, bis schließlich auch das Symplegma auf den Vasenbildern abgebildet wird. Ein Fragment des Amasis-Malers (SF 8, Abb. 5) aus Samos zeigt zwei nackte Frauen, die Schmuck und Efeukränze tragen und von Silenen eng umschlungen werden; eine der beiden hält einen Kantharos in ihrer rechten Hand. Durch die völlige Nacktheit der Frauen bringt der Amasis-Maler eine wesentliche Neuerung für seine Zeit. Ein bemerkenswertes Detail dieses Fragments ist, dass sich neben den Personengruppen eine gemalte Amphora befindet,

${ }^{27}$ Schöne 1987, 27.

${ }^{28}$ Carpenter 1986, 84 f.; Moraw 1998, 39-42.

${ }^{29}$ Schöne 1987, 28.

${ }^{30}$ A. Henrichs, Myth visualized: Dionysos and his circle in sixth-century Attic vase-painting, in: Papers on the Amasis painter and his world. J. Paul Getty Museum (Malibu 1987) 92-124. 103 f.; Isler-Kerényi 1999, 562. Die Schulterumarmung der beiden Frauen wird als erotisches Motiv gedeutet, das eine Anspielung auf die homoerotische Atmosphäre beim Symposion sein kann. Eine Bauchamphora des Amasis-Malers in Würzburg (Martin-von-Wagner-Museum L 265; attisch, um 530 v. Chr.; ABV 151, 22; Gasparri 1986, 459 Nr. 415; LIMC III 2 [1986] 346 Abb. 415 s. v. Dionysos [C. Gasparri]) zeigt zwei einander umarmende Silene. Als weibliches Gegenstück zu ihnen ist das ısapphische Paar`auf der Pariser Amphora (SF 7) zu verstehen. Vgl. Henrichs a. O. 104; Isler-Kerényi 1999, 562.

31 Stähli 1999, 216 Anm. 426; Carpenter 1986, 90.

32 Schöne 1987, 28; Carpenter 1986, 84 f. 


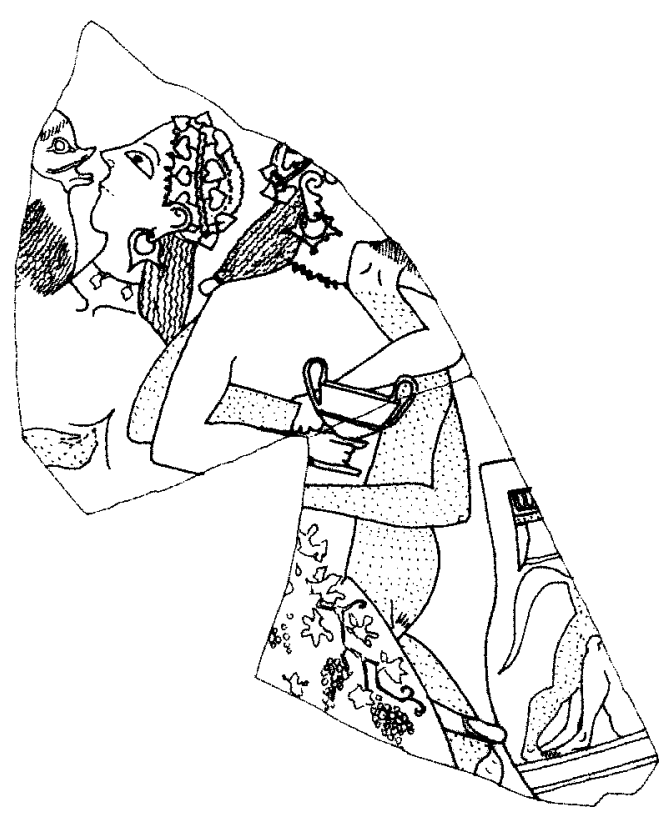

5 SF 8: Amphorafragment, Samos, Archäologisches Museum Inv. K 898

auf welcher ein ebensolches Paar in sehr engem Kontakt dargestellt ist ${ }^{33}$. Außerattische Vasenbilder dieser Zeit zeigen auch manchmal Nymphen und Silene beim direkten Geschlechtsakt ${ }^{34}$.

Die in der Periode bis 530 v. Chr. vorherrschende Darstellungsweise der Tanzbilder, die Dionysos, Silene und Nymphen beinhalten, wird in der spätschwarzfigurigen Malerei beibehalten und weist eine ständige Wiederholung dieses Themas auf einer Fülle von Vasenbildern auf, wobei nur die Anzahl der Thiasoten variiert ${ }^{35}$.

\section{Reitende Nymphen}

Ein neues Motiv der spätschwarzfigurigen Vasenbilder sind Frauen, die auf Tieren (meist auf Stieren oder Ziegenböcken) reiten, Attribute wie Efeukränze und Krotalen besitzen und manchmal gemeinsam mit Dionysos oder Silenen auftre$\operatorname{ten}^{36}$. Aufgrund der Attribute und der vegetabilen Elemente können sie als dionysische Frauen gedeutet werden.

Ein besonderes Bild aus dieser Reihe zeigt eine Lekythos in Neapel (SF 9), auf der eine nackte Frau im Herrensitz auf einem Panther reitet und in ihrer linken Hand einen Fisch hält. Die beigefügte Inschrift ergibt keinen Sinn ${ }^{37}$. Durch diese Bildformel wird das enge Verhältnis der dionysischen Frauen zu den Nereiden, den Meeresnymphen, zum Ausdruck gebracht. Es gibt Beispiele späterer Vasen, wo Fische geläufige Attribute der Nereiden sind. S. Moraw bezeichnet diese reitenden Frauen als Mänaden, die durch ihre Wesensverwandtheit mit den Nymphen eine ikonographische Angleichung erfahren ${ }^{38}$.

Die Nymphen-Mänaden-Problematik der schwarzfigurigen Vasenbilder

In der Wissenschaft ist man sich weitgehend einig, dass in der Frühzeit die Partnerinnen der Silene als Nymphen zu bezeichnen sind, wie es auch durch Inschriften belegt ist (vgl. SF 3. SF 4. SF 5). Mit dem Auftreten der dionysischen Attribute (Efeukränze, Nebris, Pardalis, Schlangen und ab der rotfigurigen Vasenmalerei auch Thyrsoi) benennen viele Wissenschaftler diese Frauen als Mänaden.

\footnotetext{
33 Carpenter 1986, 85; Moraw 1998, 42; Isler-Kerényi 1999, 561.

${ }^{34}$ Beispielsweise eine Hydria in Wien, Kunsthistorisches Museum 3577; wahrscheinlich aus Cerveteri; Adler-Maler; caeretanisch, um 530 v. Chr.; J. M. Hemelrijk, Caeretan Hydriae (Mainz 1984) 14 f. Nr. 5 Taf. 39. Krauskopf - Simon 1997,787 Nr. 54. Rückführung des Hephaistos mit Dionysos, einer Nymphe und einem Silen. Auf der Rückseite zwei Silen-NymphePaare beim Geschlechtsakt. Ferner eine Lekythos in Berlin, Antikensammlung 3243; aus Korinth; Tydeus-Maler; spätkorinthisch, 560/550 v. Chr.; D. A. Amyx, Corinthian vase-painting of the Archaic period (Berkeley 1988) 270 Nr. 4; H. Payne, Necrocorinthia. A study of Corinthian art in the archaic period (New York 1971) 324 Nr. 1372 Taf. 38, 3. 6. Mehrere SilenNymphe-Paare beim Geschlechtsakt. Ein etwas späteres Beispiel liefert die bekannte Phineus-Schale in Würzburg, Martinvon-Wagner-Museum L 164; Phineus-Maler; chalkidisch, um 520 v. Chr.; A. Rumpf, Chalkidische Vasen (Berlin - Leipzig 1927) 15 Nr. 20; LIMC VIII (1997) 896 Nr. 71 s. v. Nymphai (M. Halm-Tisserant - G. Siebert); E. Langlotz, Griechische Vasen in Würzburg (Rom 1968) Nr. 164 Taf. 26. 27. Außenfries: zwei Silen-Nymphe-Paare beim Geschlechtsakt.

35 Schöne 1987, 101-107. Eine ausführliche Zusammenstellung spätschwarzfiguriger Tanzbilder findet sich bei Schöne 1987, 278-286 Nr. 247-354.

${ }^{36}$ Moraw 1998, 46 f. Das Bildmotiv wurde genau untersucht bei: M. C. Villanueva Puig, Sur l'identité de la figure féminine assise sur un taureau dans la céramique attique à figures noires, in: C. Bérard (Hrsg.), Images et sociétés en Grèce ancienne. L'iconographie comme méthode d'analyse (Lausanne 1987) 131-139.

37 MonAnt 22, 1913, 507 (E. Gabrici).

${ }^{38}$ Moraw 1998, 50 f.
} 
Dazu muss gesagt werden, dass das kollektive Wort »Mänaden« auf keiner attischen Vase als Beischrift vorkommt, sondern aus der Dichtkunst adaptiert wurde, und zwar vor allem aus den >Bakchen $<$ des Euripides. In dieser Tragödie sind die dionysischen Frauen mit den gleichen Attributen ausgestattet, die auch auf den Vasenbildern abgebildet sind: So kam es zu der bevorzugten Namengebung der Mänaden ${ }^{39}$. Allerdings ist zu betonen, dass die Frauen in den >Bakchen Außerdem werden sie als keusch charakterisiert, was mit den schwarzfigurigen Vasenbildern nicht übereinstimmt. Mit der Betrachtung der Iliasszene, in der die Nymphen Thystla tragen, sind die dionysischen Attribute, welche zur Unterscheidung zwischen Nymphen und Mänaden dienen, kein überzeugendes Argument mehr ${ }^{40}$. Auch A. Stewart setzt sich für eine Deutung vor allem der nackten Frauen im dionysischen Gefolge als Nymphen ein, da sie keineswegs Sterbliche meinen ${ }^{41}$.

Trotzdem hat die Theorie von M. W. Edwards großen Anklang gefunden: »Nymph I use for a female figure, clothed or not, accompanying satyrs but not having any Dionysiac attribute such as the nebris, pardalis, snake or thyrsos. These are the NYФAI of the François Vase. ${ }^{42}$ G. Hedreen argumentiert dagegen, dass diese dionysischen Attribute auch für Figuren verwendet werden, die mit Sicherheit keine Mänaden sein können: so ist beispielsweise auch Hermes Träger der Nebris. Weitere Argumente gegen eine Benennung als Mänaden liefern die Namensbeischriften der dionysischen Frauen, da diese immer Namen von Nymphen tragen ${ }^{43}$. Zu den Bakchennamen schreibt C. Fränkel: »Erwartet man aber in ihren Benennungen ein Spiegelbild ihres Orgiasmus zu finden, so wird man enttäuscht: die Namen bleiben vorwiegend allgemeine Nymphen- und Frauennamen. Man nutzt den vorhandenen Namenschatz, nicht ängstlich besorgt, diese Nymphen nach dem Eigensten und Persönlichsten ihres Wesens zu benennen. Und trotz des individuellen Charakters der Mänade, den man, wie die Darstellungen lehren, stark empfand, bleibt sie doch Nymphe, so dass allgemeine Nymphennamen ihr nach wie vor zukommen. ${ }^{44}$

In der antiken Literatur sind die Nymphen und die Mänaden, die Dionysos begleiten, wesensgleich: Sie tanzen und musizieren im Gefolge des Gottes. »Die Benennung `Nympheく oder `Mänade« « ist laut S. Moraw ein »eher äußerliches und daher wandelbares Etikett, das Wesen der Betreffenden bleibt dagegen dasselbe. $\aleph^{45}$

Bedenkt man, dass das $>$ Mänadentum nur ein Zustand ${ }^{46}$ ist, in den Frauen durch den Einfluss des Gottes verfallen, so sind Nymphen davon nicht ausgeschlossen. Das stetige Gefolge des Dionysos übt auch in den $>$ Bakchen ২ des Euripides >mänadische< Tätigkeiten aus, die allerdings positive Ekstase ${ }^{47}$ bedeuten.

Die Nymphen geben sich also der dionysischen Raserei hin und können insofern als Mänaden bezeichnet werden, was meiner Meinung nach nicht bedeutet, dass sie deshalb menschliche Frauen darstellen müssen ${ }^{48}$. Wenn sich das weibliche dionysische Gefolge des Dionysos anderen Tätigkeiten als der $\mu \alpha v i \alpha$ widmet, ist der Ausdruck »Rasende« ohnedies nicht angebracht.

${ }^{39}$ Hedreen 1994, $48 \mathrm{f}$.

${ }^{40}$ Larson 2001, $94 \mathrm{f}$.

${ }^{41}$ A. Stewart, Art, desire and the body in ancient Greece (Cambridge 1997) 188 f. Seine Argumentation bezüglich der nackten Frauen beruht darauf, dass sich die menschlichen Frauen, die dionysische Rituale vollzogen, nie entkleideten.

${ }^{42}$ Edwards 1960, 80 Anm. 11.

${ }^{43}$ Hedreen 1994, 52 f. Der Name Maıvas, also die Rasende, ist zwar auch zu finden, aber bei Darstellungen, die sicher Nymphen meinen.

${ }^{44}$ C. Fränkel, Satyr- und Bakchennamen auf Vasenbildern (Halle 1912) 43.

45 Moraw 1998, 36.

${ }^{46}$ Hedreen 1994, 50.

${ }^{47}$ Krauskopf - Simon 1997, 781.

${ }^{48}$ Dieselbe Meinung wird vertreten von A. Heinemann, Bilderspiele beim Gelage. Symposiast und Satyr im Athen des 5. Jahrhunderts v. Chr., in: T. Hölscher (Hrsg.), Gegenwelten zu den Kulturen Griechenlands und Roms (München 2000) 321-349; 324 und Anm. 9-11. 


\title{
3. Die Darstellungen in der rotfigurigen Vasenmalerei
}

\author{
Ekstase und Idylle
}

Der Wahnsinn, die $\mu a v i \alpha$, ist die extremste Form der dionysischen Ekstase und wird in der rotfigurigen Vasenmalerei bevorzugt dargestellt.

Auf frührotfigurigen Gefäßen bis ca. 470 v. Chr. werden die dionysischen Frauen fast ausschließlich als Rasende im Thiasos präsentiert ${ }^{49}$, wozu sich natürlich besonders gut die Weiterführung der vor allem im spätschwarzfigurigen Stil beliebten Tanzbilder eignet. Das wohl bekannteste und qualitätsvollste Gefäß ist die Spitzamphora des Kleophrades-Malers in München (RF 1): In der Mitte ist Dionysos zu sehen, der Weinrebe und Kantharos in den Händen hält und mit geneigtem Kopf in tanzender Bewegung dargestellt ist. Er wird von zwei Nymphen umgeben, die jeweils einen Thyrsos halten und sich mit dessen Hilfe der aufdringlichen Silene erwehren. Die Linke hat ein Rehfell umgehängt und hält eine Schlange in ihrer Hand ${ }^{50}$. Die Silene sind unter den Henkeln dargestellt und bei frontaler Betrachtung der Vase gar nicht zu sehen, woraus sich ergibt, dass Dionysos und sein weibliches Gefolge ein näheres Verhältnis haben, bei welchem die Silene als störend empfunden werden, ja sogar Außenseiter sind ${ }^{51}$. Auf der anderen Seite des Gefäßes ist ein flötenspielender Silen frontal abgebildet. Er wird von zwei Nymphen umgeben, die ihre Köpfe weit in den Nacken zurückgeworfen und ihre Münder geöffnet haben, die sich ihrer Ekstase somit völlig hingeben $^{52}$. Der Silen und die Nymphen streben in ihren Bewegungen auseinander und nehmen keinerlei Bezug auf ihre Umgebung. Im Gegensatz zu den schwarzfigurigen Tanzbildern, bei welchen Silene und Nymphen in fröhliches Treiben vertieft waren, hat sich die Interaktion bei den rotfigurigen Vasenbildern zu einem rituellen $\operatorname{Tanz}^{53}$ gewandelt und das friedliche, nahe Verhältnis zwischen den weiblichen und männlichen Dionysosbegleitern hat sich aufgelöst. Außerdem sind bei vielen Darstellungen die Nymphen den Silenen in der Anzahl überlegen ${ }^{54}$. Die mit dem Einsetzen der rotfigurigen Vasenmalerei einhergehenden beträchtlichen Veränderungen in der Ikonographie manifestieren sich deutlich in neuen Elementen, die Ausdruck der Ekstase sind ${ }^{55}$; dies sind: der Thyrsos ${ }^{56}$, zurückgeworfene oder gesenkte Köpfe, offene Haare, Flügelärmel ${ }^{57}$ und das Motiv der Silene abwehrenden Nymphen.

Die vehemente Abwehr durch die dionysischen Frauen erklärt sich mit dem engen Verhältnis zwischen Dionysos und seinen Anhängerinnen und der Konzentration der Frauen auf die Ekstase ${ }^{58}$. Häufig wird von einer konsequenten Verwandlung von Nymphen in Mänaden gesprochen, da die bei Euripides beschrie-

\footnotetext{
49 Schöne 1987, 113; Moraw 1998, 52 f. 82.

${ }_{50}$ Moraw 1998, 82; Schöne 1987, 148.

${ }^{51}$ McNally 1978, 120.

52 Schöne 1987, 148; Moraw 1998, 82.

53 McNally 1978, 121.

${ }_{54}$ Moraw 1998, 56. 112.

55 Edwards 1960, 85 f.

${ }^{56}$ Der Thyrsosstab ist ein neues Element, das erst mit der rotfigurigen Vasenmalerei um 520 v. Chr. einsetzt. Gefertigt wurde der Thyrsos aus dem Stängel der Narthexstaude, wobei an der Spitze ein Efeubüschel befestigt wurde. Der Stab wurde erstmals von Oltos dargestellt und setzt sich rasch als kanonisches dionysisches Element durch. Den Frauen wird dadurch eine spezielle Kraft verliehen und ihre Naturverbundenheit ausgedrückt bzw. hervorgehoben. Das Tragen von Pflanzen (Efeuzweige und Weinranken) ist seit der Mitte des 6. Jhs. v. Chr. ein wesentliches Charakteristikum für die dionysische Ikonographie. Der Thyrsos ist wohl eine künstlich gefertigte Weiterentwicklung dieser Pflanzen. Es gibt in der attisch-schwarzfigurigen Vasenmalerei jedoch bereits Vorformen des echten Thyrsos, deren Stäbe aus einem Stamm mit längeren Efeuzweigen und einem Efeubüschel in der Mitte bestanden (vgl. auch das Wort $\theta \dot{v} \sigma \theta \lambda \alpha$ bei Homer - s. o.). Deshalb muss es schon vor 520 v. Chr. einen Kult gegeben haben, bei welchem ein Kultstab mit Efeupflanzen Verwendung fand. Vgl. dazu I. Krauskopf, Thystla, Thyrsoi und Narthekophoroi. Anmerkungen zur Geschichte des dionysischen Kultstabes, Thetis 8, 2001, 47-52; Moraw 1998, 53 f.; Schöne 1987, 146; McNally 1978, 121.

${ }^{57}$ Der englische Ausdruck »wing-sleeves« wurde von J. D. Beazley geprägt und ist die Bezeichnung für die durch das Gewand verhüllten Arme der dionysischen Frauen, die den Eindruck erwecken, es seien Flügel. s. J. D. Beazley, A hydria by the Kleophrades Painter, AntK 1, 1958, 6.

${ }^{58}$ McNally 1978, 120; Schöne 1987, 135.
} 
benen Mänaden keusch sind und sich die dionysischen Frauen auf den ekstatischen Vasenbildern auch derart verhalten $^{59}$. Mögliche Erklärungen für die Veränderungen in der Ikonographie könnten ein neuer mythologischer Stoff oder die Entstehung eines neuen Mysterienkultes zu dieser Zeit sein. Obwohl die Bilder sicher mythologische Themen erzählen, könnten die Vasenmaler trotzdem von reellen Kultpraktiken beeinflusst worden $\operatorname{sein}^{60}$. Zu bedenken ist allerdings immer, dass sich diverse Attribute und Darstellungsmodi auch mit dem Zeitgeschmack und den künstlerischen Fähigkeiten bzw. Erfindungen der Vasenmaler ändern.

In der Folgezeit zwischen 470 und

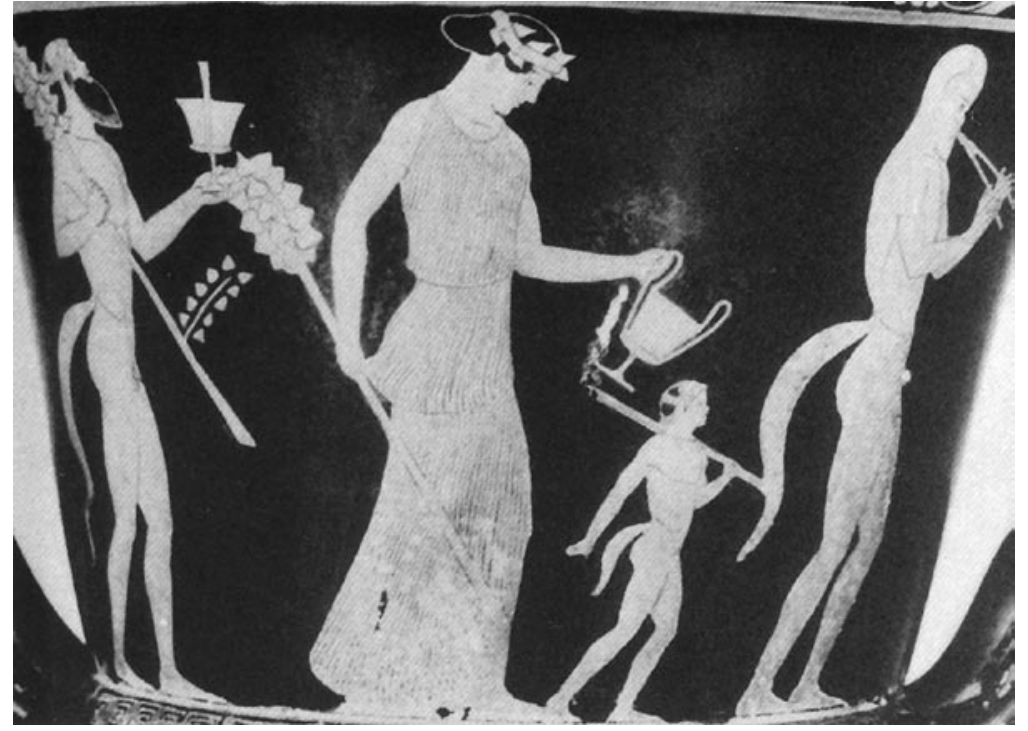

6 RF 3: Kelchkrater, Karlsruhe, Badisches Landesmuseum 208 (B3) 430 v. Chr. werden die Darstellungen rasender Frauen zwar weitergeführt, den prozentuell größten Anteil am dionysischen Repertoire stellen allerdings Nymphen in idyllischen Naturlandschaften oder sog. verbürgerlichte Darstellungen derselben ${ }^{61}$. Ein gutes Beispiel liefert ein Glockenkrater des Lykaon-Malers in Warschau (RF 2): In der Mitte des Bildes ist Dionysos zu sehen, der zu einem Silen blickt, welcher gerade Wein in einen Glockenkrater gießt. Neben ihm steht eine Nymphe, die ihr rechtes Bein auf einen Felsen aufgestützt hat, ihre Haare fallen offen auf die Schultern herab und ihr Blick geht in die Ferne. Nahe bei ihr sitzt ein flötenspielender Silen. Rechts neben Dionysos steht ein Reh, das gerade von einer Nymphe gestreichelt wird, der paradoxerweise der Name MAINA $\Sigma$, also die Rasende, beigegeben ist, obwohl sie ganz friedlich dargestellt ist ${ }^{62}$. Hier wird eine idyllische Phantasiewelt evoziert, wobei die Nymphen in eine liebevoll zärtliche Rolle schlüpfen. Die nun abgebildeten harmlosen Tiere wie Rehe oder Hasen betonen das friedliche Leben der Nymphen in der Natur. Bei diesen Szenen werden die Silene nicht mehr an den Rand gedrängt, sondern beide Geschlechter stehen wieder gleichberechtigt nebeneinander ${ }^{63}$. Auch Bilder von Satyrfamilien, bestehend aus Nymphe, Silen und Satyrkind, treten zu dieser Zeit auf: Ein Kelchkrater des Villa-Giulia-Malers in Karlsruhe (RF 3, Abb. 6) zeigt eine derartige Satyrfamilie bei einer Prozession. Voran schreitet der weißhaarige Großvater, der den Doppelaulos spielt, gefolgt von einem Silen-Kind, das eine brennende Fackel über der Schulter trägt. Dahinter schreiten eine Nymphe mit Thyrsos und Kantharos in Händen, den Kopf gesenkt haltend, und ein Silen mit den gleichen Attributen, der aber den Kopf in den Nacken geworfen hat ${ }^{64}$. F. Brommer erklärt die Vermenschlichung der Silene und Nymphen, die jetzt Vater- und Mutterfiguren übernehmen und sich in ihrer Charakterisierung sehr von den ekstatisch schwärmenden Thiasoten unterscheiden, durch das Aufkommen des Satyrspiels ${ }^{65}$. Ich schließe mich aber der Meinung von A. Schöne an, dass die Ursache des Aufkommens dieser Bilder wohl im Zeitgeist der Polis zu suchen ist, da auch auf nichtdionysischen Vasenbildern die Entwicklung zu intimeren, familiären Szenen festzustellen is ${ }^{66}$. Bei allen Darstellungen von

\footnotetext{
${ }^{59}$ Stähli $1999,178$.

${ }^{60}$ Edwards 1960, 84-87; Schöne 1987, 159 f. Eine andere Deutungsmöglichkeit wird durch das Aufkommen des Satyrspiels in Betracht gezogen: Die Silene werden als lächerliche Gestalten charakterisiert, was in den Vasenbildern durch die Dominanz und Überlegenheit der dionysischen Frauen zum Ausdruck kommt. Vgl. dazu Schöne 1987, 190; Moraw 1998, 116; Larson 2001, 95.

${ }^{61}$ Moraw 1998, $58 \mathrm{f}$.

${ }^{62}$ J. D. Beazley, Greek vases in Poland (Oxford 1928) $54 \mathrm{f}$.

${ }^{63}$ Moraw 1998, 59. 89. 121.

${ }^{64}$ F. Brommer, Satyrspiele. Bilder griechischer Vasen (Berlin 1944) 33; Schöne 1987, 166; Moraw 1998, 123.

${ }^{65}$ Brommer (Anm. 64) $33 \mathrm{f}$.

${ }^{66}$ Schöne 1987, 86.
} 


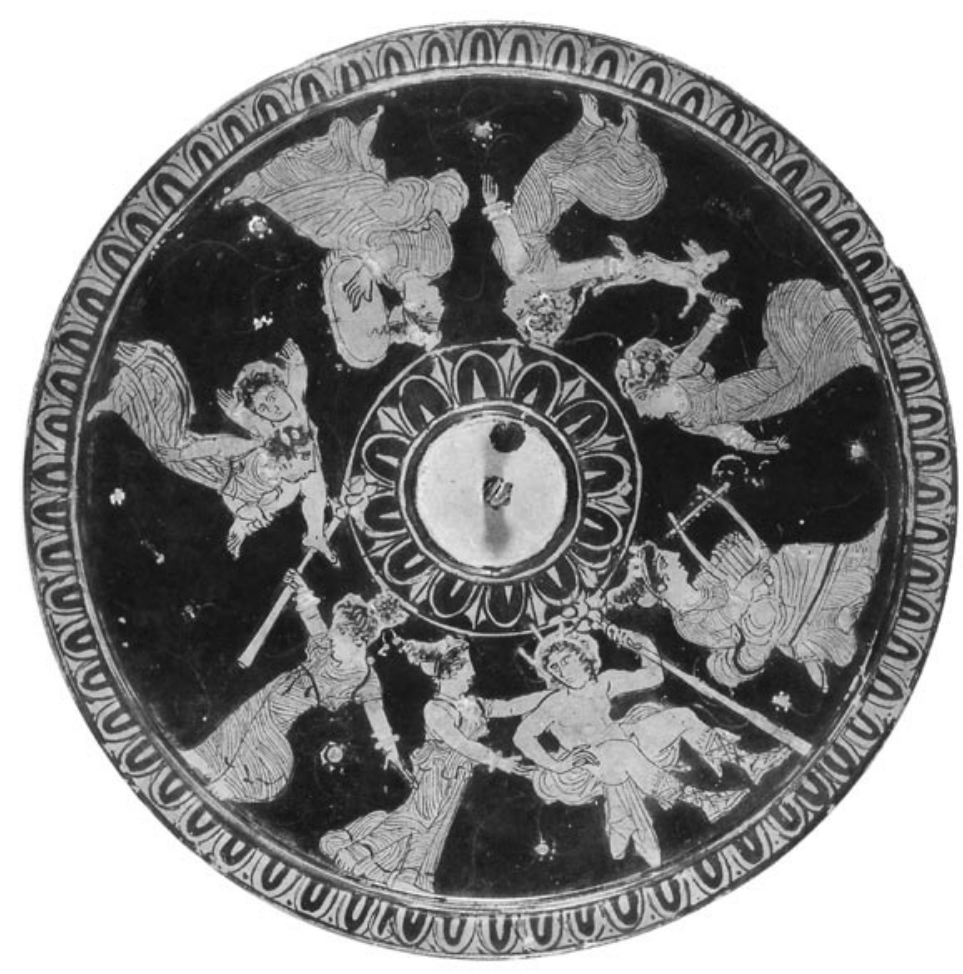

7 RF 4: Pyxis, London, British Museum E 775

Satyrfamilien, Libations- und Prozessionsszenen ist eine /Verbürgerlichung` der dionysischen Frauenfiguren zu beobachten. Oft sind ihnen keinerlei Attribute beigegeben, sodass sie sich nicht mehr von gleichzeitigen Darstellungen Athener Bürgerinnen unterscheiden ${ }^{67}$.

Es ist also festzuhalten, dass das Verhältnis zwischen den weiblichen und männlichen Thiasoten auch in der rotfigurigen Vasenmalerei nicht immer feindlich ist, sondern es im Gegenteil sogar zu liebevollen Familiendarstellungen kommt und zahlreiche Beispiele des sidyllischen Thiasos` die Bezeichnung »Rasende« für die Frauen innerhalb dieses Geschehens in Frage stellen.

Im Reichen Stil leben Tanzbilder ekstatischen Charakters wieder auf, wobei die meisten Bilder Rückgriffe auf frührotfigurige Darstellungsmodi zeigen. Eine Neuerung dieser Zeit bilden jedoch die Darstellungen der verderblichen, also der negativen Raserei ${ }^{68}$ : So zeigt beispielsweise eine Pyxis in London vom Ende
, die den sitzenden Dionysos umgeben. des 5. Jahrhunderts v. Chr. (RF 4, Abb. 7) sieben rasende Frauen, die den sitzenden Dionysos umgeben.
Zwei von ihnen sind im Begriff, ein Reh zu zerreißen, eine andere hat ein Kind über die Schulter geworfen; Silene sind nicht anwesend. Gegen Ende des 5. Jahrhunderts v. Chr. gibt es mehrere Darstellungen dieser Art: Die von Dionysos gesandte Raserei wird mit allen Schrecken erregenden Auswirkungen gezeigt und die Mänade als grausames, übernatürliches Wesen geschildert ${ }^{69}$. Die Uraufführung der euripideischen >Bakchen fand um 404 v. Chr. statt, weswegen man hier wahrscheinlich mit einem Einfluss des Dramas rechnen kann. Die Vasenmaler wurden von dem neuen Thema, der verderblichen Raserei als Strafe des Gottes, inspiriert und meinen mit diesen Bildern menschliche Mänaden.

\section{Das Kampfmotiv}

In der frührotfigurigen Vasenmalerei taucht das Kampfmotiv zwischen Nymphe und Silen erstmals auf einer nikosthenischen Amphora des Oltos (RF 5) auf: Die Seite A des Gefäßes zeigt einen Silen, der eine riesenhafte Nymphe attackiert, indem er sich in gebückter Haltung an sie klammert und sie dabei ihren rechten Arm ausstreckt. Auf Seite B hingegen hat die Nymphe den Angreifer bereits zu Boden gebracht, packt ihn am Schopf und lässt eine Schlange auf ihn los. Das Darstellungsmuster eines gebückten Silens, der eine aufrecht stehende Nymphe umklammert, knüpft an Kampfdarstellungen zwischen Peleus und Thetis $a^{70}$.

\footnotetext{
${ }^{67}$ Moraw 1998, $59 \mathrm{f}$.

${ }^{68}$ Schöne 1987, 151.

${ }^{69}$ Moraw 1998, 97-99. 262.

${ }^{70}$ Schöne 1987, 134; Moraw 1998, 114. Ein gutes Vergleichbeispiel stellt eine Schale des Peithinos dar: Berlin, Antikensammlung F 2279, attisch, 510/500 v. Chr.; ARV² 115, 2; CVA Berlin, Antikenmuseum (2) Taf. 61, 1. Peleus und Thetis im Kampf. Peleus umklammert Thetis' Taille. Sie streckt ihren rechten Arm aus, welche Geste bereits auf der Amphora des Oltos beobachtet wurde. Thetis wird im Kampf von einem kleinen Löwen und mehreren Schlangen unterstützt.
} 
Parallelen zwischen den Kampfgruppen sind die Beigabe von Tieren und die Überlegenheit der Frauen im Kampf, was durch ihre Körpergröße zusätzlich betont wird. Die der Thetis beigegebenen wilden Tiere drücken allerdings auch ihre Verwandlungsfähigkeit aus. Ferner scheint die Nereide auf den Vasenbildern die Überlegene zu sein, obwohl sie im Mythos verliert ${ }^{71}$. Die Begleitpersonen der Thetis sind meist Nereus und die Nereiden (Meeresnymphen), die an ihren Namensbeischriften identifiziert werden können ${ }^{72}$. Das sog. Verfolgungsmotiv, wie es bei Darstellungen von Nymphen und Silenen seit dem 6. Jahrhundert v. Chr. bekannt ist, wird auch für Peleus und Thetis verwendet. Interessanterweise zeigen zahlreiche Gegenseiten der Peleus-Thetis-Darstellungen dionysische Bildmotive, und die Nereiden bewegen sich sehr ähnlich zu den schwärmenden Nymphen, wodurch die Verwandtschaft der beiden Frauentypen gut zum Ausdruck $\mathrm{kommt}^{73}$.

\section{Die Übergabe des Dionysoskindes}

Laut literarischer Überlieferung wird die Mutter des Dionysos, Semele, von Zeus' göttlicher Übermacht getötet, nachdem sie ihn gebeten hatte, sich in seiner wahren Gestalt (in Blitz und Donner) zu zeigen. Zeus rettet das ungeborene Kind und näht es sich in seinen Schenkel ein, um es von dort neu zu gebären. Danach wird der Dionysosknabe zu den Nymphen von Nysa gebracht, von diesen genährt und aufgezogen ${ }^{74}$.

»Zwar hat das gewaltige Meer seinen männlichen Beherrscher, und die kraftvoll einherwandelnden Ströme ihre Götter. Aber selbst in der Tiefe und auf dem Spiegel der See sind die Meermädchen und Meeresgöttinnen wichtiger als die männlichen Geister, und Nereus ist nicht von Söhnen, sondern von Töchtern umgeben. Die Quellen aber, die Seen und die feuchten Niederungen gehören ganz den weiblichen Geistern. Dort wohnt auch Artemis, die Schöne, die aller Geburt ans Licht hilft oder die Frauen in bitteren Wehen sterben läßt. Sie ist die Pflegerin der Kinder. Und so sind alle Mädchen der Feuchte, die man Nymphen, d. h. junge Frauen oder Bräute, nennt - ein Wort, das bei den Italikern in der Form lympha das Wort Wasser bedeutet - Wärterinnen. « $^{75}$

In der Vasenmalerei taucht das Thema der Dionysosübergabe an die nysäischen Nymphen erst um 480 v. Chr. auf und wird im Wesentlichen nur bis ca. 420 v. Chr. fortgeführt ${ }^{76}$. Bei diesen Darstellungen sind die dionysischen Frauen aufgrund der literarischen Überlieferung sicher als Nymphen zu bezeichnen: Ein Stamnos in Paris (RF 6) zeigt Zeus und eine in einen langen Mantel gehüllte Nymphe, die einander gegenüberstehen. Die Nymphe hat das Kind bereits entgegengenommen, der kleine Dionysos wendet sich aber noch zu seinem Vater um. Die zweite Nymphe, die hinter der ersten sitzt, hält einen Thyrsos und eine Phiale in Händen, wodurch sie als zum dionysischen Gefolge zugehörig ausgewiesen wird. Sie thront vor einem ionischen Gebäude, welches wohl den Übergabeort bei einem Heiligtum andeuten soll und die Feierlichkeit des Geschehens unterstreicht ${ }^{77}$.

Ein Volutenkrater des Altamura-Malers in Ferrara (RF 7, Abb. 8) zeigt als Mittelgruppe wiederum Zeus, mit einem Szepter ausgestattet, eine lang gewandete Nymphe und das Kind, das die Nymphe gerade aufnimmt; in der Linken hält das Kind einen kleinen Kantharos. Die Mittelszene wird von zwei Nymphen flankiert, wobei die linke einen kleinen Panther in der Hand hält, welcher sich mit seinen Vorderbeinen auf

\footnotetext{
${ }^{71}$ McNally 1978, 118; Moraw 1998, 114 f. mit Anm. 492.

${ }^{72}$ X. Krieger, Der Kampf zwischen Peleus und Thetis in der griechischen Vasenmalerei. Eine typologische Untersuchung (Münster 1975) $88 \mathrm{f}$.

${ }^{73}$ Krieger (Anm. 72) $114 \mathrm{f}$.

${ }^{74}$ Schefold 1981, 27-29.

75 Otto $1960,156$.

${ }^{76}$ Schöne 1987, 85. E. H. Loeb hat bei der Betrachtung der Bilder, die das Thema in jeder Periode unterschiedlich darstellen, drei verschiedene chronologische Gruppen herausgearbeitet: 1. In der Zeit bis 460 v. Chr. übergibt Zeus das Kind den Nymphen von Nysa. 2. Von 460 bis zur Jahrhundertwende bringt Hermes das Kind zu einer Nymphe oder dem Papposilen. Später kann Hermes von einer Nymphe oder einem Silen abgelöst werden. 3. Nach der Wende vom 5. zum 4. Jh. v. Chr. wird der Knabe vorwiegend zusammen mit göttlichen Wesen dargestellt, die ihm Trauben reichen. s. bei E. H. Loeb, Die Geburt der Götter in der griechischen Kunst der klassischen Zeit (Jerusalem 1979) 39.

${ }^{77}$ Gasparri 1986, 481 Nr. 703; Schöne 1987, 82.
} 


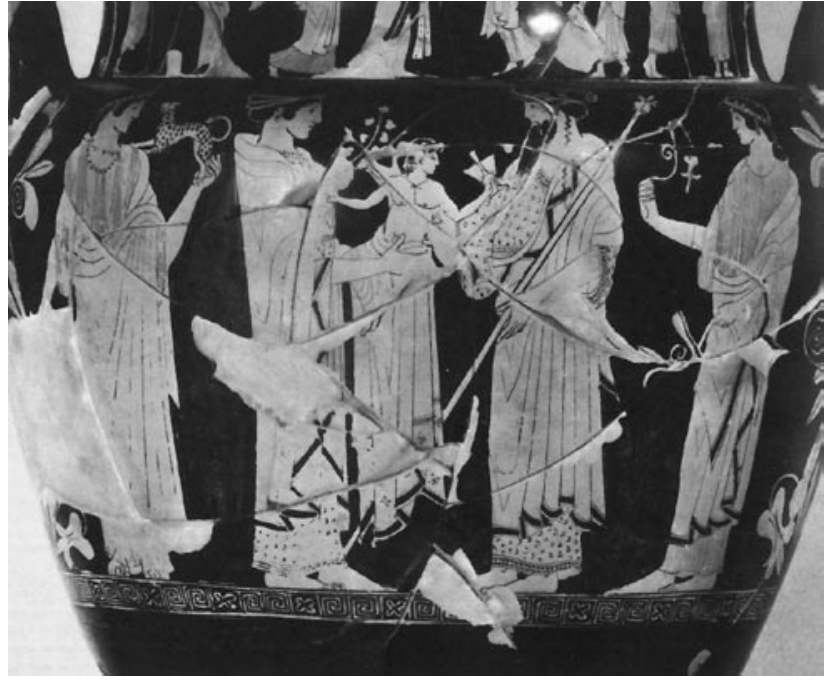

8 RF 7: Volutenkrater, Ferrara, Museo Nazionale 2737 ihrer Schulter abstützt. Efeuzweige, Ranken und der Panther verdeutlichen die dionysische Sphäre ${ }^{78}$. A. Schöne spricht in diesem Zusammenhang von einer Angleichung von Nymphen und Mänaden, da die nysäischen Nymphen mit >Mänadenattributen ausgestattet werden ${ }^{79}$. E. Simon sieht hingegen keine unterschiedlichen Identitäten bei den Ammen des Dionysos und seinem späteren göttlichen Gefolge: Die Nymphen von Nysa begleiten ihren Zögling später als Mänaden ${ }^{80}$. Mit der Betrachtung der Bilder, welche die Dionysosübergabe thematisieren, fällt auf, dass die nysäischen Nymphen die gleichen Attribute wie das dionysische Gefolge bei ekstatischen Tanzszenen erhalten. Daraus ergibt sich, dass nicht alle als dionysisch gekennzeichneten Frauen automatisch als sterbliche Mänaden bezeichnet werden können.

\section{Der Tod des Pentheus}

Bei ekstatischen Bildern ist häufig nicht zu entscheiden, ob Nymphen oder Mänaden dargestellt sind. Es gibt allerdings Darstellungen vom Tod des Pentheus, bei denen die Frauen aufgrund der literarischen Überlieferung sicher als Mänaden gedeutet werden können.

In den >Bakchen des Euripides findet sich die ausführlichste Beschreibung dieses Mythos: Pentheus, der König von Theben, möchte die Mänaden, die sich in den Bergen dem dionysischen Rausch hingeben, zurückholen und zur Vernunft bringen. Auch ihm wird von Dionysos Wahnsinn gesandt, sodass er als Mänade verkleidet die Thebanerinnen in den Bergen beobachtet. Seine Mutter Agaue führt den eksatischen Schwarm der Mänaden an und gemeinsam mit den anderen Frauen reißt sie ihren Sohn in Stücke, im Glauben, es sei ein Löwe ${ }^{81}$.

Die früheste Darstellung dieses Themas zeigt ein fragmentarisch erhaltener Psykter des Euphronios in Boston (RF 8): Zwei Mänaden reißen am bereits abgetrennten Oberkörper des bärtigen Pentheus, neben dessen Kopf sein Name beigeschrieben ist. Weitere Mänaden sind mit einem Bein und anderen Körperteilen des Pentheus dargestellt ${ }^{82}$. Zu beachten sind die Thyrsoi, mit welchen die Mänaden ausgestattet sind, und die wallenden Haare der abgebildeten Frauen.

Eine apulische Patera aus der Mitte des 4. Jahrhunderts v. Chr. (RF 9, Abb. 9) zeigt die Szene in ganz anderer Weise: Pentheus, der nur mit einer Chlamys bekleidet, aber mit Schwert und Speeren bewaffnet ist, wird von je zwei rasenden Mänaden umgeben, die mit Thyrsos oder Schwert bewaffnet sind und ihn angreifen ${ }^{83}$.

\footnotetext{
78 Schefold 1981, 31; Gasparri 1986, 481 Nr. 702.

${ }^{79}$ Schöne 1987, 82.

${ }^{80}$ E. Simon, Opfernde Götter (Berlin 1953) 48.

${ }^{81}$ J. R. March, Euripides' Bakchai: A reconsideration in the light of vase-paintings, BICS 36, 1989, 33-65; 35. Aufgrund des vermehrten Auftretens derartiger Darstellungen zwischen 510 und 490 v. Chr. muss hinter diesen Bildern eine ältere Version des Mythos vermutet werden. Von Aischylos ist ebenfalls eine Pentheus-Tragödie bezeugt, die in den Jahren nach $480 \mathrm{v}$. Chr. aufgeführt wurde. Die frührotfigurigen Vasenbilder geben wohl eine noch frühere Version des Mythos wieder. Vgl. dazu Schefold 1981, 181-186; Schöne 1987, 67.

82 Schefold 1981, 181 f.; Schöne 1987, 67.

${ }^{83}$ LIMC VII (1994) 308 Nr. 10 s. v. Pentheus (J. Bazant - G. Berger-Doer). Auch wenn man erwarten würde, dass die Bilder des Reichen Stils und des 4. Jhs. v. Chr. von Euripides beeinflusst wären, so stellt sich bei der Betrachtung der Vasenbilder das Gegenteil heraus: Pentheus tritt nie in Frauenkleidern auf und nirgends wird Agaue als Mörderin ihres Sohnes inschriftlich identifiziert. Vgl. March (Anm. 81) 36-43. 50-52; Schöne 1987, 70.
} 


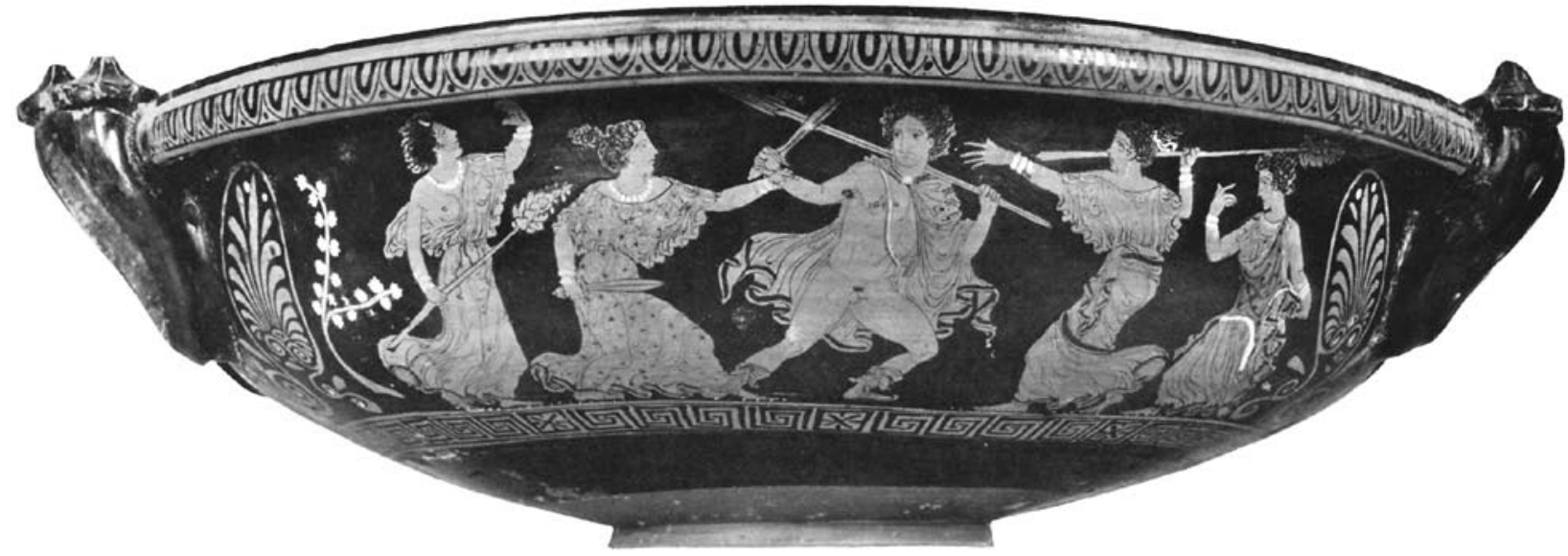

9 RF 9: Patera, Ruvo, Jatta Msueum J 1617

Das Handeln der Mänaden bei Euripides gipfelt in der Zerreißung des Pentheus, was den Höhepunkt der von Dionysos geschickten $\mu \alpha v i \alpha$ darstellt ${ }^{84}$. Das Aufkommen der Bilder im frührotfigurigen Stil lässt sich möglicherweise auch auf die Beliebtheit ekstatischer Vasenbilder in dieser Zeit zurückführen ${ }^{85}$. Bei der Betrachtung der Mänaden kann man erkennen, dass sie sich in keiner Weise von tanzenden Nymphen unterscheiden: Sie tragen Thyrsoi, haben oft wallendes Haar und tanzen ekstatisch. Die Mänaden sind, wie die Nymphen auch, durchweg als dionysische Frauen gekennzeichnet. Aber da sie hier mit verderblicher Raserei bestraft werden, wie dies den Thebanerinnen in den >Bakchen $<$ des Euripides widerfährt, sind sie von den lieblichen Nymphen zu unterscheiden.

\section{Die Lenäenvasen}

Neben dem von Euripides beschriebenen Mänadenkult existierte auch ein realer historischer Kult: Eine lokale Chronik aus Magnesia am Mäander berichtet von einer Kultvereinigung, welche die `Bakchen des Euripides als Inspirationsquelle für ihre kultischen Handlungen nahm und den Sparagmos (das Zerreißen wilder Tiere mit bloßen Händen) oder die Omophagia (das Verschlingen rohen Fleisches) durch die Darbringung von Opfern nachspielte. Diese historischen Mänaden haben folglich das Verhalten der mythischen Mänaden in symbolische Formen umgestaltet ${ }^{86}$. Die verschiedenen Kultreigen veranstalteten im Namen des Gottes Tänze und vollzogen bestimmte Kulthandlungen: Beispielsweise galt es, das Dionysoskind in seiner Wiege aufzuwecken. Die Frauen des Mythos (in diesem Fall die nysäischen Nymphen) waren Vorbild für diverse Kultgemeinschaften ${ }^{87}$. In diesem Zusammenhang sind die sog. Lenäenvasen zu erwähnen, welche hauptsächlich zwischen 490 und 420 v. Chr. entstehen. Ihre Bilder zeigen immer eine Maske des Dionysos, die meist an einer Säule befestigt ist. Um das Idol tanzen in heftigen Bewegungen ausschließlich Frauen, die oft eine Hand zum Idol des Dionysos erheben ${ }^{88}$. Das wohl berühmteste und qualitativ beste Werk ist eine Schale des Makron in Berlin (RF 10, Abb. 10): Mehrere Frauen tanzen ekstatisch um das Dionysosidol und

${ }^{84}$ Henrichs 1994, $37 \mathrm{f}$.

${ }^{85}$ Moraw 1998, 148. Auffallend ist die Ähnlichkeit der Darstellungsweise mit Bildern der Hochklassik und des Reichen Stils, auf denen dionysische Frauen Tiere zerreißen. Vgl. dazu Schöne 1987, 69.

${ }^{86}$ Stähli 1999, 185-190, vgl. auch Moraw 1998, 18-21. Mit dem Phänomen der historischen Mänaden beschäftigt sich ausführlich: R. S. Kraemer, Women's devotion to Dionysos, in: Her share of the blessings. Women's religions among Pagans, Jews and Christians in the Greco-Roman world (New York 1992) 36-49.

${ }^{87}$ Otto $1960,158 \mathrm{f}$.

${ }^{88}$ H. Killet, Zur Ikonographie der Frau auf attischen Vasen archaischer und klassischer Zeit (Berlin 1994) 137; Schöne 1987, 173; Moraw 1998, 74. 


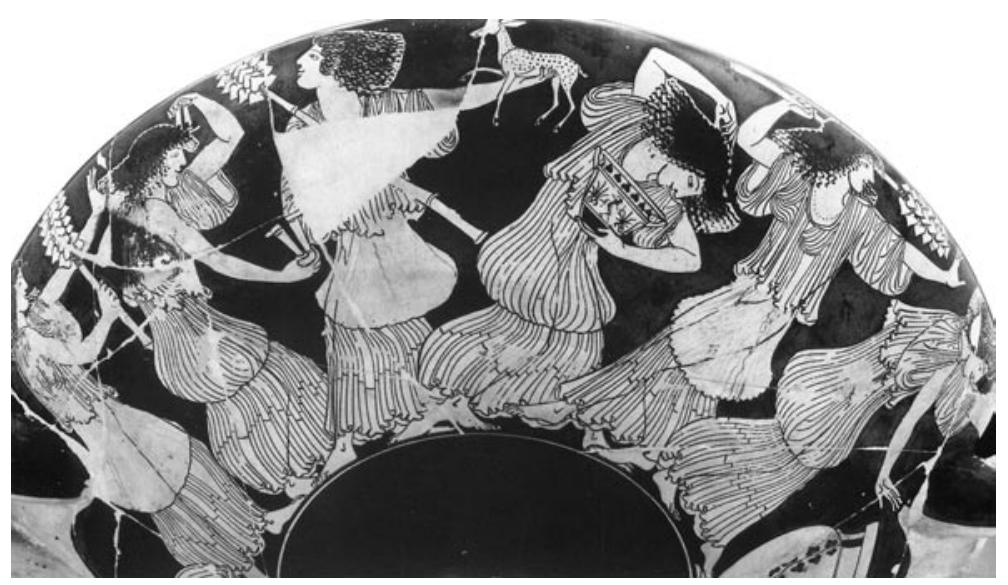

10

RF 10: Schale, Berlin, Antikensammlung F 2290

einen Altar, wobei manche mit Thyrsoi ausgestattet sind, eine die Doppelflöte spielt, eine andere Krotalen in Händen hält, und auch Reh und Skyphos als Attribute vorkommen ${ }^{89}$.

Angesichts der großen Übereinstimmung in der Gestaltung der Dionysosmaske auf allen diesen Vasen und der einheitlichen Form der ekstatischen Frauen, die das Bild verehren, geht man von der Darstellung eines dionysischen Festes, eventuell der Lenäen, aus. Nun scheint es logisch, dass diese Frauen historische Mänaden sind, die Dionysos bei seinem großen Fest verehren ${ }^{90}$.

Auch bei diesen Bildern ist festzustellen, dass die abgebildeten Frauen dionysische Attribute aufweisen und sich in keiner Weise von den mythischen Begleiterinnen des Dionysos, Nymphen oder Mänaden, unterscheiden.

\section{Die Nymphen-Mänaden-Problematik der rotfigurigen Vasenbilder}

Mit dem Aufkommen der Vasenbilder ekstatischen Charakters und der damit einhergehenden Abneigung dionysischer Frauen gegenüber den Silenen stellt sich erneut die Frage, ob sich die Nymphen nun in Mänaden sverwandelt haben. Nachdem die Nymphen um die Mitte des 6. Jahrhunderts v. Chr. mit dionysischen Attributen ausgestattet wurden, vollzieht sich am Ende des 6. Jahrhunderts ein neuerlicher Wandel: Ekstatisch tanzende Frauen, die häufig die Angriffe der Silene abwehren, werden zum dominanten Element in der dionysischen Ikonographie.

Die plötzliche `Keuschheit` der Frauen wurde oftmals zum Anlass genommen, die Frauen nun als Mänaden zu benennen ${ }^{91}$, zumal sie in den $>$ Bakchen des Euripides als keusch beschrieben werden, während die Nymphen laut literarischer Überlieferung die Gefährtinnen der Silene sind. Es muss allerdings dagegen gehalten werden, dass das Verhältnis der Nymphen zu den Silenen am Anfang des 6. Jahrhunderts v. Chr. ja auch nicht friedlich war, sondern das Verfolgungsmotiv hier seinen Ursprung hatte. J. Larson hält einen Einfluss des Satyrspiels am Ende des 6. Jahrhunderts v. Chr. für möglich: Die lächerliche Figur des unzivilisierten Satyrs komme besser zum Ausdruck, wenn er von seiner Partnerin unterdrückt wird und seine sexuellen Wünsche unerfüllt bleiben ${ }^{92}$. T. H. Carpenter konstatierte mehrmals, dass es sich bei den dionysischen Frauen der rotfigurigen Vasenbilder um halbgöttliche Nymphen handelt, und es keinen Grund gibt, die Frauen als Sterbliche anzusehen. Die Argumentation beruht hauptsächlich auf den Bildern der Dionysosübergabe, wo sicher Nymphen dargestellt werden, diese aber ebenso mit `Mänadenattributen< ausgestattet werden ${ }^{93}$.

Die Analyse der Vasenbilder hat ergeben, dass zwar in der frührotfigurigen Malerei die dionysische Raserei das Hauptthema der Darstellungen ist, sich das Bild in der Klassik allerdings drastisch verändert. Vermehrt treten Szenen auf, in denen der Thiasos ruhig und friedlich dargestellt wird (Libationen, Prozes-

\footnotetext{
${ }^{89}$ Schöne 1987, 175.

${ }^{90}$ Killet (Anm. 88) 138. 144.

${ }^{1}$ McNally 1978, 129 f.; Stähli 1999, 175 und Anm. 415; Hedreen 1994, 65: Er argumentiert, dass die Nymphen mit ihren neuen Attributen und ihrem geänderten Verhalten zu »soldiers of Dionysos« werden und damit den Bakchen des Euripides am nächsten stehen.

${ }_{92}$ Larson 2001, 95.

${ }_{93}$ Carpenter 1997, 52-62; T. H. Carpenter, Nymphs, not maenads, on Attic red-figure vases, AJA 99, 1995, 314.
} 
sionen und Satyrfamilien), wodurch die Abneigung gegenüber den Silenen zurückgeht und aggressive Angriffe seitens der Silene nur noch vereinzelt vorkommen. Gerade die Existenz friedlicher Satyrfamilien ist ein Beweis dafür, dass die Frauen den Silenen keineswegs immer abgeneigt sind. Außerdem wurde festgestellt, dass es in jeder Epoche Darstellungen gibt, welche die Zuneigung der Nymphen zu ihren Partnern visualisieren.

Vasenbilder ekstatischen Charakters erscheinen im Reichen Stil wieder vermehrt. Die Bilder zeigen Ausmaße des destruktiven Wahnsinns ${ }^{94}$, ihre Entstehungszeit fällt in die Zeit der Uraufführung der >Bakchen des Euripides. Deshalb glaube ich die Vasenbilder von diesem Drama beeinflusst, womit sich ergibt, dass die Frauen dieser grausamen Darstellungen menschliche Mänaden sind, welche als Strafe des Gottes die negative Ekstase erleben.

Die Verwandtschaft der dionysischen Frauen mit den Nereiden, den Meeresnymphen, lässt sich mehrfach bezeugen. Zum einen zeigt die bereits betrachtete Lekythos in Neapel (SF 9), auf der eine reitende Frau einen Fisch hält, die Wesensverwandtheit von dionysischen Frauen und Nereiden, welche auch im Mythos belegt ist: Die Mutter des Dionysos, Semele, hat drei Schwestern - Ino, Agaue und Autonoe. In der »Odyssee« ist Ino eine Seegöttin, Agaue und Autonoe gehören dem Kreis der Nereiden an ${ }^{95}$. Dadurch erklärt sich das enge Verhältnis der mit Dionysos verbundenen Frauen zu den Wasser- und Meeresnymphen und legt eine Deutung der dionysischen Frauen als Nymphen nahe. Zum anderen weisen Kampfbilder mit Umklammerungsmotiv (RF 5) eine starke Parallele zu den Kampfdarstellungen zwischen Peleus und der Meeresnymphe Thetis auf. Die dadurch ausgedrückte Verwandtschaft zwischen Thetis und den dionysischen Frauen spricht ebenso für eine Deutung als Nymphen, und zwar auch derjenigen, die sich gegen ihre Angreifer behaupten.

\section{Ergebnisse}

Aus den eben konstatierten Feststellungen ergibt sich meiner Meinung nach, dass dionysische Frauen nicht aufgrund ihrer Attribute einem bestimmten Frauentypus zuzuordnen sind, sondern dass aus dem Kontext geschlossen werden muss, ob göttliche Nymphen, mythische oder historische Mänaden gemeint sind. Klar ist die Deutung bei den Bildern der Dionysosübergabe: Es sind dies die nysäischen Nymphen, die Ammen des Dionysos. Bei den Lenäenvasen handelt es sich um den Vollzug historischer Mänadenkulte. Die Bilder der Zerreißung des Pentheus zeigen mythische Mänaden, die sich gegen ihren Willen im dionysischen Gefolge befinden und mit verderblicher Raserei bestraft werden, was in der Ermordung des Pentheus gipfelt. Die sich im idyllischen Thiasos befindlichen Frauen sind Nymphen, ebenso die Frauen der Satyrfamilien. Bei manchen Bildern des Reichen Stils tritt die verderbliche Raserei in den Vordergrund, was die Frauen als Mänaden kennzeichnet. Dass es sicher Überschneidungen der Frauentypen gab, zeigen die Darstellungen, die das Zerreißen von Opfertieren thematisieren, da hier enge Parallelen zu den Pentheus-Bildern zu beobachten sind. Die frührotfigurigen Ekstasebilder meinen wohl eher Nymphen, die gemeinsam mit ihrem Gott durch die Wälder schwärmen und sich der positiven Raserei hingeben. Diese können insofern als Mänaden bezeichnet werden, als sie in den Zustand mänadischer Raserei verfallen. Allerdings befinden sie sich stetig und freiwillig im dionysischen Gefolge. Manchmal zeigen die Bilder ausschließlich Frauen, die sich der Ekstase hingeben. Sind die Silene gänzlich abwesend, könnte es sich auch um mythische Mänaden handeln.

Welcher Gruppe die jeweils dargestellten Frauen angehören, ist also häufig aus dem Bildzusammenhang zu erschließen. Bei einigen Bildmotiven, besonders bei den ekstatischen Tänzen, kommt es allerdings zu Überschneidungen und eine sichere Deutung muss offenbleiben.

\footnotetext{
${ }^{94}$ Henrichs 1994, 37.

${ }^{95}$ Otto $1960,157 \mathrm{f}$.
} 


\section{Katalog}

\section{Schwarzfigurige Vasen}

\section{SF 1}

Spitzaryballos Brindisi 1669; aus Brindisi

Spät-protokorinthisch, ca. 650 v. Chr.

Lit.: Isler-Kerényi 1991, 296. 306 Abb. 8; D. A. Amyx, Corinthian vase-painting of the Archaic period (Berkeley 1988) 333. 659.

Beschreibung: Ein ithyphallischer Mann (Vorläufer eines Silens) geht mit einer Keule auf eine von im abgewendete Frau los, die ein langes Gewand trägt und mit einem Hasen spielt.

\section{SF 2}

Lekythos Buffalo, Albright Gallery G 600 Art des Gorgo-Malers; attisch, 600-580 v. Chr. Lit.: ABV 12, 22; Moraw 1998, Taf. 1 Abb. 1.

Beschreibung: Ein Silen reitet auf einem Maultier, welches einer fliehenden Nymphe in den Arm beißt.

\section{SF 3}

Dinos London, British Museum 1971.1 1-1 1

Sophilos; attisch, 580/570 v. Chr.

Lit.: Beazley Para. 19, 16; Carpenter 1986, Taf. 1 A.

Beschreibung: Drei an der Prozession für Peleus und Thetis teilnehmende Frauen, die inschriftlich als NYФAI bezeichnet werden. Beim Pferdegespann von Ares und Aphrodite fünf Frauen mit der Beischrift MO $\Sigma$ AI, die mittlere spielt die Syrinx.

\section{SF 4}

Abb. 3

Dinos Athen, Nationalmuseum 15165 (Acr. 587); von der Akropolis

Sophilos; attisch, 580/570 v. Chr.

Lit.: ABV 39, 15; LIMC VIII (1997) 903 Nr. 2 s. v. Nysa I, Nysai (U. Gottschall); Carpenter 1986, Taf. 3 A.

Beschreibung: Drei Frauenköpfe mit der Beischrift NY $\Sigma$ AI, die Frau en face spielt die Syrinx.

\section{SF 5}

Volutenkrater Florenz, Museo Archeologico Etrusco 4209; aus Chiusi

Kleitias; attisch, 570/560 v. Chr.

Lit.: ABV 76, 1; LIMC VIII (1997) 894 Nr. 25 s. v. Nymphai (M. Halm-Tisserant - G. Siebert); Isler-Kerényi 1999, 558 Abb. 2-4.

Beschreibung: Silene und Nymphen im Gefolge des Dionysos bei der Rückführung des Hephaistos, die Frauen tragen die Beischrift NYФAI. Eine Nymphe wird von einem Silen getragen, die anderen tanzen und haben Musikinstrumente bei sich.
SF 6

Abb. 4

Kolonettenkrater New York, The Metropolitan Museum of Art 31.11.11

Lydos; attisch, um 550 v. Chr.

Lit.: ABV 108, 5; Gasparri 1986, 471 Nr. 563; Moraw 1998, Taf. 3 Abb. 8 a-d.

Beschreibung: Lebhaftes Thiasostreiben bei der Rückführung des Hephaistos. Alle Frauen tragen die Nebris, eine hat eine Schlange umgelegt.

\section{SF 7}

Halsamphora Paris, Cabinet des Médailles 222; aus Vulci Amasis-Maler; attisch, um 540 v. Chr.

Lit.: ABV 152, 25; Beazley Para. 63; Gasparri 1986, 451 Nr. 294; Carpenter 1986, Taf. 17.

Beschreibung: Dionysos und zwei einander umarmende Frauen stehen einander gegenüber. Eine der Frauen hat als besonderes Attribut das Pantherfell umgehängt und trägt einen Hirsch in der Hand.

SF 8

Abb. 5

Fragmente einer Amphora Samos, Archäologisches Museum Inv. K 898; aus dem Heraion von Samos Amasis-Maler; attisch, 550/540 v. Chr.

Lit.: ABV 151, 18; S. Karouzou, The Amasis Painter (Oxford 1956) Taf. 30 Abb. 2. 3.

Beschreibung: Zwei nackte, aber Schmuck tragende Frauen, die von Silenen eng umschlungen werden. Eine gemalte Amphora mit einer ebensolchen Darstellung.

\section{SF 9}

Lekythos Neapel, Museo Archeologico Nazionale 86382; aus Cumae Sappho-Maler; attisch, Six-Technik, um 500 v. Chr. Lit.: E. Haspels, Attic Black-figured Lekythoi (Paris 1936) 227, 41; Krauskopf - Simon 1997, 789 Nr. 86; MonAnt 22, 1913, Taf. 61, 3.

Beschreibung: Eine nackte Frau reitet auf einem Panther und hält in ihrer Linken einen Fisch. Aneinanderreihung von Buchstaben, die aber keinen Sinn ergibt. 


\section{Rotfigurige Vasen}

\section{RF 1}

Spitzamphora München, Antikensammlung 2344; aus Vulci Kleophrades-Maler; attisch, um 500 v. Chr.

Lit.: ARV ${ }^{2}$ 182, 6; Krauskopf - Simon 1997, 786 Nr. 36; Schöne 1987, Taf. 22. 23; Moraw 1998, Taf. 10 Abb. 28 a-d. Beschreibung: Der tanzende Dionysos wird umgeben von zwei Nymphen, welche die Annäherungen der Silene abwehren. Zwei weitere Frauen, die durch das Flötenspiel eines Silens in Ekstase verfallen sind.

\section{RF 2}

Glockenkrater Warschau, Nationalmuseum 142355

Lykaon-Maler; attisch, um 440 v. Chr.

Lit.: ARV² 1045, 6; Carpenter 1997, Taf. 27 A.

Beschreibung: Dionysos wird umgeben von einem Silen, der eine Weinamphora leert und einer in sich versunkenen Nymphe. Auf der anderen Seite befindet sich eine Nymphe, die ein Reh streichelt.

RF 3

Abb. 6

Kelchkrater Karlsruhe, Badisches Landesmuseum 208 (B3); aus Agrigent

Villa-Giulia-Maler; attisch, um 450 v. Chr.

Lit.: ARV² 618, 3; Schöne 1987, Taf. 28, 2.

Beschreibung: Prozession einer Satyrfamilie. Weißhaariger, flötenspielender Silen, gefolgt von einem Silen-Kind, einer Nymphe und einem weiteren Silen.

\section{RF 4}

Pyxis London, British Museum E 775; aus Eretria

Abb. 7

Art des Meidias-Malers; attisch, 410/400 v. Chr.

Lit.: ARV ${ }^{2}$ 1328, 92; Krauskopf - Simon 1997, 785 Nr. 33; Moraw 1998, Taf. 23 Abb. 57.

Beschreibung: Sieben rasende Mänaden umgeben den sitzenden Dionysos, wobei zwei ein Reh zerreißen und eine weitere ein Kind über die Schulter geworfen hat.

\section{RF 5}

Nikosthenische Amphora Paris, Musée du Louvre G 2; aus Etrurien

Oltos; attisch, um 520 v. Chr.

Lit.: ARV ${ }^{2}$ 53, 2; Krauskopf - Simon 1997, 788 Nr. 62; Schöne 1987, Taf. 21.

Beschreibung: Seite A: Ein Silen attackiert eine Nymphe. Seite B: Eine Nymphe hat den angreifenden Silen zu Boden gebracht und lässt eine Schlange auf ihn los.

\section{RF 6}

Stamnos Paris, Musée du Louvre G 188; aus Vulci Maler der Florentiner Stamnoi; attisch, um 470/460 v. Chr. Lit.: ARV ${ }^{2}$ 508, 1; Gasparri 1986, 481 Nr. 703; LIMC III 2 (1986) 380 Abb. 703 s. v. Dionysos (C. Gasparri).

Beschreibung: Eine Nymphe hat das Dionysoskind bereits von Zeus entgegengenommen, es wendet sich aber noch zu seinem Vater um. Eine weitere Nymphe sitzt dahinter, Thyrsos und Phiale in Händen.

RF 7

Abb. 8

Volutenkrater Ferrara, Museo Nazionale 2737; aus Spina Altamura-Maler; attisch, um 470/460 v. Chr.

Lit.: ARV² 589, 3; Beazley Para. 393; Gasparri 1986, 481 Nr. 702; Schefold 1981, 31 Abb. 23.

Beschreibung: Eine Nymphe ist gerade im Begriff, den Dionysosknaben entgegenzunehmen, dieser streckt einen Arm nach ihr aus, blickt aber zu Zeus zurück. Zwei weitere Nymphen flankieren das Geschehen, die linke mit einem kleinen Panther am Arm.

\section{RF 8}

Fragmente eines Psykters Boston, Museum of Fine Arts 10.221; aus Orvieto

Euphronios; attisch, 520/510 v. Chr.

Lit.: ARV 2 16, 14; 1619; Beazley Para. 321; LIMC VII (1994)

312 Nr. 39 s. v. Pentheus (J. Bazant - G. Berger-Doer); Moraw 1998, Taf. 8 Abb. 25 a-b.

Beschreibung: Mehrere Mänaden mit Teilen des zerrissenen Körpers des Pentheus. Oberhalb seines Kopfes ist sein Name beigeschrieben.

\section{RF 9}

Abb. 9

Patera Ruvo, Jatta Museum J 1617; aus Ruvo Gruppe von Oxford 269; apulisch, 360/350 v. Chr.

Lit.: A. D. Trendall - A. Cambitoglou, The red-figured vases of Apulia I. Early and middle Apulian (Oxford 1978) 400, 27; LIMC VII 1 (1994) 308 Nr. 10 s. v. Pentheus (J. Bazant - G. Berger-Doer), LIMC VII 2 (1994) 251 Abb. 10 s. v. Pentheus (J. Bazant - G. Berger-Doer).

Beschreibung: Pentheus ist mit einem Schwert und zwei Speeren bewaffnet. Zu seinen Seiten vier Mänaden, die ihn attackieren.

\section{RF 10}

Abb. 10

Schale Berlin, Antikensammlung F 2290; aus Vulci Makron; attisch, 490/480 v. Chr.

Lit.: ARV² 462, 48; Gasparri 1986, 427 Nr. 41; Moraw 1998, Taf. 13 Abb. $34 \mathrm{a}-\mathrm{b}$.

Beschreibung: Mehrere Mänaden tanzen ekstatisch um ein Dionysosidol, das neben einem Altar steht. Thyrsoi, Skyphoi und Rehe sind ihnen als Attribute beigegeben. 


\begin{tabular}{|c|c|}
\hline \multicolumn{2}{|r|}{ Abgekürzt zitierte Literatur } \\
\hline Carpenter 1986 & $\begin{array}{l}\text { T. H. Carpenter, Dionysian Imagery in archaic Greek art. Its development in black-figure vase- } \\
\text { painting (Oxford 1986). }\end{array}$ \\
\hline Carpenter 1997 & T. H. Carpenter, Dionysian Imagery in Fifth-Century Athens (Oxford 1997). \\
\hline Edwards 1960 & M. W. Edwards, Representation of maenads on archaic red-figure vases, JHS 80, 1960, 78-87. \\
\hline Gasparri 1986 & C. Gasparri in: LIMC III (1986) 414-514 s. v. Dionysos. \\
\hline Hedreen 1994 & G. Hedreen, Silen, nymphs and maenads, JHS 114, 1994, 47-69. \\
\hline Henrichs 1994 & $\begin{array}{l}\text { A. Henrichs, Der rasende Gott: Zur Psychologie des Dionysos und des Dionysischen in Mythos und } \\
\text { Literatur, AuA 40, 1994, 31-58. }\end{array}$ \\
\hline Isler-Kerényi 1991 & $\begin{array}{l}\text { C. Isler-Kerényi, Dionysos: Dio delle donne? Iconografia dionisiaca II, in: F. Berti (Hrsg.), Diony- } \\
\text { sos. Mito e Mistero (Ferrara 1991) 293-303. }\end{array}$ \\
\hline Isler-Kerényi 1999 & C. Isler-Kerényi, Frauen um Dionysos vom 7. Jahrhundert bis um 540 v. Chr., AA 1999, 553-566. \\
\hline Krauskopf - Simon 1997 & I. Krauskopf - E. Simon in: LIMC VIII (1997) 780-803 s. v. Mainades. \\
\hline Larson 2001 & J. Larson, Greek Nymphs. Myth, Cult, Lore (New York 2001). \\
\hline McNally 1978 & S. McNally, The maenad in early Greek art, Arethusa 11, 1978, 101-135. \\
\hline Moraw 1998 & $\begin{array}{l}\text { S. Moraw, Die Mänade in der attischen Vasenmalerei des 6. und 5. Jh. v. Chr. Rezeptionsästheti- } \\
\text { sche Analyse eines antiken Weiblichkeitsentwurfs (Mainz 1998). }\end{array}$ \\
\hline Otto 1960 & F. W. Otto, Dionysos. Mythos und Kultus (Tübingen 1960). \\
\hline Schefold 1981 & K. Schefold, Die Göttersage in der klassischen und hellenistischen Kunst (München 1981). \\
\hline Schöne 1987 & $\begin{array}{l}\text { A. Schöne, Der Thiasos. Eine ikonographische Untersuchung über das Gefolge des Dionysos in der } \\
\text { attischen Vasenmalerei des 6. und 5. Jhs. v. Chr. (Göteborg 1987). }\end{array}$ \\
\hline Stähli 1999 & A. Stähli, Die Verweigerung der Lüste. Erotische Gruppen in der antiken Plastik (Berlin 1999). \\
\hline
\end{tabular}

Mag. Gabriele Schmidhuber

Universität Salzburg, FB Altertumswissenschaften, Klassische und Frühägäische Archäologie, Residenzplatz 1, A-5020 Salzburg

E-Mail: ariadni@gmx.at

Abbildungsnachweis: Abb. 1: nach Isler-Kerényi 1991, 306 Abb. 8; Abb. 2: nach Isler-Kerényi 1991, 305 Abb. 5; Abb. 3: nach Carpenter 1986, Taf. 3 A; Abb. 4: nach K. Schefold, Götter- und Heldensagen der Griechen in der spätarchaischen Kunst (München 1978) 32 Abb. 24; Abb. 5: nach C. Isler-Kerényi, Civilizing Violence. Satyrs on 6th-century Greek vases (Freiburg 2004) 65 Abb. 32; Abb. 6: nach Carpenter 1997, Taf. 25 A; Abb. 7: nach LIMC VIII 2 (1997) 529 Nr. 33 s. v. Mainades (I. Krauskopf - E. Simon); Abb. 8: nach LIMC III 2 (1986) 380 Nr. 702 s. v. Dionysos (C. Gasparri); Abb. 9: nach H. Sichtermann, Griechische Vasen in Unteritalien aus der Sammlung Jatta in Ruvo (Tübingen 1966) Taf. 139; Abb. 10: nach Carpenter 1997, Taf. 21. 